\title{
COMPRESSIBILIDADE DE SOLOS VERSUS INTENSIDADE DE TRÁFEGO EM UM POMAR DE LARANJA E PISOTEIO ANIMAL EM PASTAGEM IRRIGADA
}

\section{CLÁUDIA LIANE RODRIGUES DE LIMA}

Tese apresentada à Escola Superior de Agricultura

“Luiz de Queiroz”, Universidade de São Paulo, para obtenção do título de Doutor em Agronomia, Área de Concentração: Solos e Nutrição de Plantas.

P I R A C I C A B A

Estado de São Paulo - Brasil

Janeiro - 2004 


\title{
COMPRESSIBILIDADE DE SOLOS VERSUS INTENSIDADE DE TRÁFEGO EM UM POMAR DE LARANJA E PISOTEIO ANIMAL EM PASTAGEM IRRIGADA
}

\author{
CLÁUDIA LIANE RODRIGUES DE LIMA \\ Engenheiro Agrícola
}

Orientador: Prof. Dr. ALVARO PIRES DA SILVA

\begin{abstract}
Tese apresentada à Escola Superior de Agricultura
“Luiz de Queiroz”, Universidade de São Paulo, para obtenção do título de Doutor em Agronomia, Área de Concentração: Solos e Nutrição de Plantas.
\end{abstract}

P I R A C I C A B A

Estado de São Paulo - Brasil

Janeiro - 2004 
Dados Internacionais de Catalogação na Publicação (CIP)
DIVISÃO DE BIBLIOTECA E DOCUMENTAÇÃO - ESALQ/USP

Lima, Cláudia Liane Rodrigues de Compressibilidade de solos versus intensidade de tráfego em um pomar de laranja e pisoteio animal em pastagem irrigada / Cláudia Liane Rodrigues de Lima. - Piracicaba, 2004.

60 p. : il

Tese (doutorado) - Escola Superior de Agricultura Luiz de Queiroz, 2004.

Bibliografia.

1.Fisíca do solo 2. Laranja 3. Pastagens 4. Pomares 5. Propriedade física do solo I. Título

CDD 631.43

\section{"Permitida a cópia total ou parcial deste documento, desde que citada a fonte - O autor"}




\section{AGRADECIMENTOS}

Ao professor Alvaro Pires da Silva pela orientação, ensinamentos, amizade e incentivo durante o curso.

Aos colegas de pós graduação Herdjania Veras de Lima, Ricardo Romero, Silvia Imhoff, Tairone Paiva Leão e aos técnicos do Departamento de Solos e Nutrição de Plantas, Flávia Morales, Jair da Silva, Nancy Amaral e Wladimir Rosignolo pela amizade e auxílio na realização deste trabalho.

À minha família pelo carinho.

À Coordenação do Curso de Solos e Nutrição de Plantas da ESALQ/USP pela oportunidade de realizar este curso.

À Fundação Coordenação de Aperfeiçoamento de Pessoal de Nível Superior (CAPES) pela concessão da bolsa de estudo. 


\section{SUMÁRIO}

Página

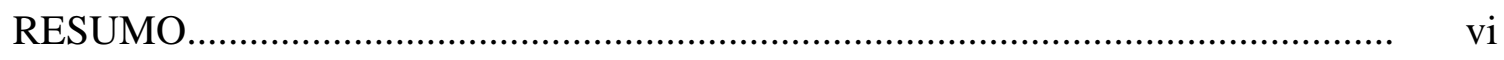

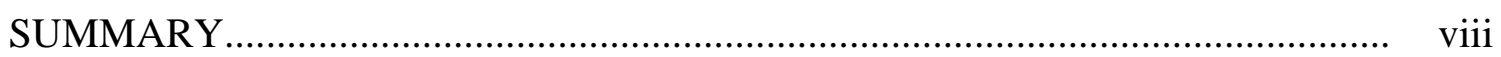

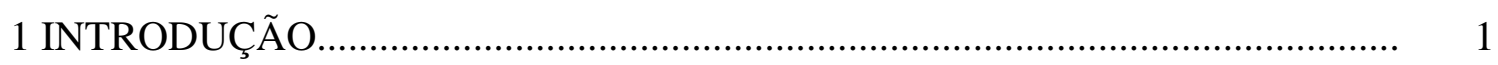

2 REVISÃO DE LITERATURA.......................................................................

3 HETEROGENEIDADE DA COMPACTAÇÃO DE UM LATOSSOLO VERMELHO AMARELO SOB POMAR DE

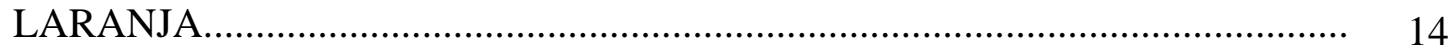

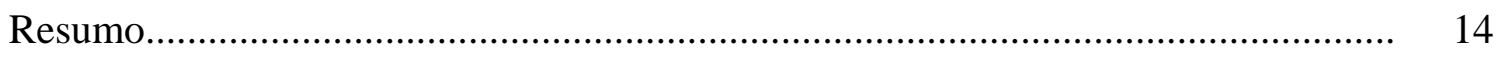

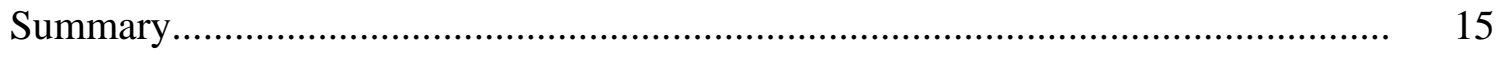

3.1 Introdução.................................................................................................... 15

3.2 Material e Métodos........................................................................................ 17

3.3 Resultados e Discussão....................................................................................... 19

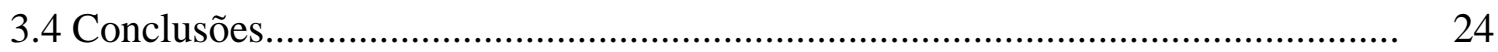

4 COMPRESSIBILIDADE DE UM ARGISSOLO VERMELHO SOB SISTEMAS DE PASTEJO ROTACIONADO INTENSIVO IRRIGADO E NÃO RRIGADO...................................................................................... 25

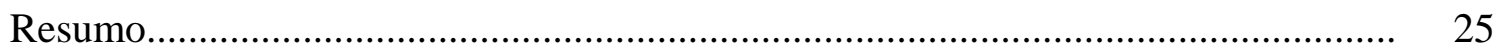

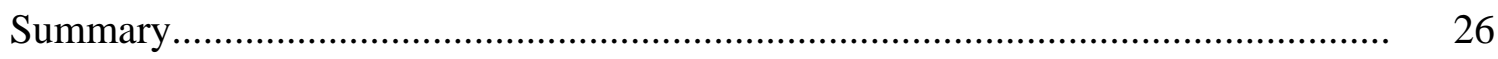

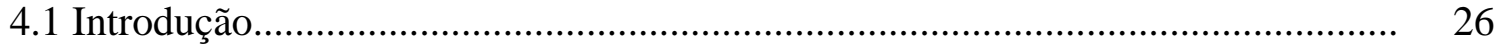

4.2 Material e Métodos............................................................................................ 28

4.3 Resultados e Discussão................................................................................... 30

4.4 Conclusões.............................................................................................. 36

5 ESTIMATIVA DA CAPACIDADE DE SUPORTE DE CARGA DO SOLO A PARTIR DA AVALIAÇÃO DA RESISTÊNCIA À PENETRAÇÃO................... 38 




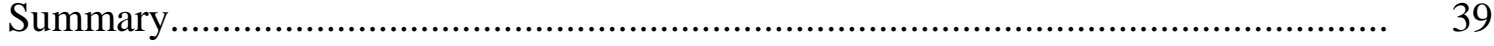



5.2 Material e Métodos.................................................................................... 41

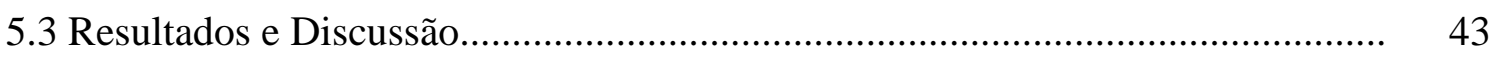

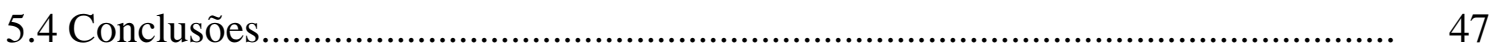

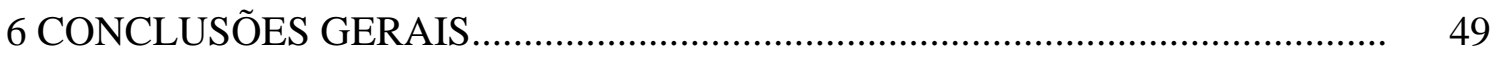

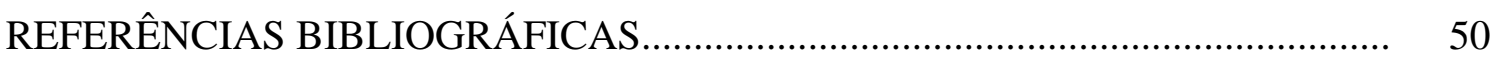




\title{
COMPRESSIBILIDADE DE SOLOS VERSUS INTENSIDADE DE TRÁFEGO EM UM POMAR DE LARANJA E PISOTEIO ANIMAL EM PASTAGEM IRRIGADA
}

\author{
Autora: CLÁUDIA LIANE RODRIGUES DE LIMA \\ Orientador: Prof. Dr. ALVARO PIRES DA SILVA
}

\section{RESUMO}

Nos últimos anos, novas estratégias têm sido desenvolvidas na obtenção de incrementos da produtividade agrícola associados à manutenção da qualidade do solo. No entanto, as terras agrícolas vêm perdendo a sua qualidade física decorrente dos processos de compactação a que estão sendo expostas. A compactação do solo, destacase a nível mundial como um dos principais responsáveis pela redução da produtividade das culturas. Pesquisas têm descrito o processo de compressão de solos formados sob a influência de clima temperado, bem como modelos que relacionam esse processo com os atributos intrínsecos dos solos. A informação disponível também demonstra que o processo de compactação depende do sistema de manejo de solo adotado. Entretanto, existe a necessidade de desenvolver estudos que permitam uma melhor compreensão do processo de compactação de solos desenvolvidos sob clima tropical. Nesse sentido, este trabalho testou a hipótese de que o processo de compactação e os indicadores derivados da curva de compressão de solos tropicais são influenciados diferenciadamente pelo sistema de manejo aplicado, devido às alterações que o mesmo induz nas propriedades 
físicas do solo. O objetivo geral deste estudo foi avaliar quantitativamente o processo de compactação e os indicadores “índice de compressão" e "pressão de preconsolidação" de solos submetidos a diferentes intensidades de tráfego em um pomar de laranja e diferentes intensidades de pisoteio animal em uma pastagem irrigada e obter, se possível, relações que permitam estimar esses indicadores a partir de propriedades do solo facilmente mensuráveis. Os objetivos específicos foram: i) determinar a influência de diferentes intensidades de tráfego no processo de compactação e nos parâmetros índice de compressão e pressão de preconsolidação de um Latossolo Vermelho Amarelo sob pomar de laranja e, a partir desses indicadores, avaliar a heterogeneidade da compactação; ii) avaliar a influência do pisoteio animal na compressibilidade de um Argissolo Vermelho submetido aos sistemas de pastejo rotacionado intensivo: irrigado e não irrigado; e iii) obter funções que permitam estimar a capacidade de suporte de carga do solo a partir de uma propriedade facilmente mensurável e de extrema importância para o crescimento de plantas: a resistência do solo à penetração. Os resultados referentes ao primeiro objetivo indicaram que o processo de compactação foi influenciado pela intensidade de tráfego, visto que os valores de pressão de preconsolidação diferiram nas posições de amostragem entre rodado, rodado e projeção da copa em relação à linha de cultivo. A pressão de preconsolidação resultou ser um indicador mais sensível que o índice de compressão na avaliação da heterogeneidade da compactação em áreas citrícolas. Os resultados obtidos no segundo objetivo revelaram diferenças na compressibilidade do solo, refletindo-se nas curvas de compressão uniaxial dos sistemas de pastejo rotacionado intensivo irrigado e não irrigado. Os sucessivos ciclos de pastejo induziram aumento da pressão de preconsolidação do solo nos dois sistemas de pastejo, sendo significativamente superior no sistema de pastejo rotacionado intensivo irrigado. No que se refere ao terceiro objetivo, foi determinada uma relação significativa, positiva e linear entre a pressão de preconsolidação e a resistência do solo à penetração medida no laboratório e no campo. Estes resultados possibilitam afirmar que a capacidade de suporte de carga do solo pode ser estimada a partir de uma propriedade física facilmente mensurável como a resistência do solo à penetração avaliada no campo. 


\title{
SOIL COMPRESSIBILITY VERSUS TRAFFIC INTENSITY IN AN ORANGE ORCHARD AND ANIMAL TRAMPLING IN AN IRRIGATED PASTURE
}

\author{
Author: CLÁUDIA LIANE RODRIGUES DE LIMA \\ Adviser: Prof. Dr. ALVARO PIRES DA SILVA
}

\section{SUMMARY}

In the last years new strategies have been developed to obtain increment of the agricultural productivity maintaining the soil quality. However, the agricultural lands quality is decreasing as a consequence of the soil compaction process. Soil compaction is considered as one of the main factors responsible for the reduction of the cultures productivity. Researches related to soils formed under the influence of temperate climate have described the process of soil compression, and developed models that relate this process with the soil intrinsic attributes. Studies have also shown that the soil compression process depends on the soil management practices. However, it is still necessary to develop studies that allow a better understanding the compaction process of soils developed under tropical climate. In this context, this research tested the hypothesis that the soil compaction process is influenced differently by the management practices, due to the alterations that induce on the soil physical properties. The general objective of this study was to evaluate quantitatively the compaction process, and the indicators "compression index" and "preconsolidation pressure” of soils submitted to different traffic intensities in an orchard, and different animal trampling intensities in an irrigated short duration grazing system, and to obtain relationships that allow to estimate these 
indicators using easily measurable soil attributes. The specific objectives were: $i$ ) to determinate the influence of different traffic intensities in the soil compaction process, and in the compression index and preconsolidation pressure of an Haplustox under an orange orchard, and to use these indicators to evaluate the soil compaction heterogeneity; ii) to evaluate the influence of the animal trampling on the soil compressibility of an Hapludalf submitted to two rotation short-duration grazing systems: not irrigated and irrigated; and iii) to obtain functions that allow to estimate the load support capacity using an easily measurable and very important soil property for plant growth; i.e. the soil resistance to penetration. The results related to the first objective indicated that the compaction process was influenced by the traffic intensity, since that the preconsolidation pressure values were different between the sampling positions; i.e., between wheel track, wheel track, canopy projection, and row. The preconsolidation pressure demonstrated to be a more sensitive indicator than the compression index in the evaluation of the soil compaction heterogeneity in an orange orchards. The results related to the second objective have indicated differences in the soil compressibility, which in turn were expressed in the soil compression curves of the irrigated and not irrigated rotation short-duration grazing systems. The successive grazing cycles have induced increases of the soil preconsolidation pressure values of the two grazing systems, being significantly greater in the irrigated rotation short-duration grazing system. The results related to the third objective have demonstrated the existence of a significant, positive and linear relationship between the preconsolidation pressure values and the soil mechanical resistance that were measured in the laboratory and in the field. These results make possible to state that the soil load support capacity can be estimated using an easily measurable soil property; i.e. the soil resistance to penetration. 


\section{INTRODUÇÃO}

O incremento da produtividade agrícola é condicionado pela interação entre vários fatores como tipo de clima, cultura e solo. O solo condiciona, por meio de suas propriedades físicas, o crescimento das raízes das plantas e, decorrente disso, a produtividade das culturas. Diferentes técnicas têm sido utilizadas no intuito de se obter aumento da produção agrícola, como adubação, irrigação e a adoção de diferentes sistemas de manejo. Entretanto, se estima que cerca de 68 milhões de hectares de terras agrícolas estão perdendo a sua qualidade física decorrente dos processos de compactação

a que estão sendo expostas. Assim, a compactação destaca-se a nível mundial como um dos principais entraves na obtenção de elevadas produtividades.

O termo compactação refere-se ao processo que descreve o decréscimo de volume de solos não saturados quando uma determinada pressão externa é aplicada no solo, seja por máquinas agrícolas, equipamentos de transporte ou animais. O entendimento do processo de compactação do solo baseia-se na avaliação das curvas de compressão uniaxial. Esta curva é representada graficamente pela relação entre algum parâmetro relacionado à estrutura do solo (razão de vazios ou densidade do solo) com o logaritmo da pressão aplicada, e permite estimar as alterações que podem ocorrer na qualidade estrutural do solo. A partir da curva de compressão uniaxial pode-se obter os parâmetros chamados índice de compressão e pressão de preconsolidação, os quais têm sido amplamente aceitos como indicadores da susceptibilidade do solo à compactação e de sua capacidade de suporte de carga, respectivamente. Ambos os indicadores são influenciados pelas propriedades físico-mecânicas dos solos, sendo a umidade, a textura, o teor de carbono orgânico e o estado de compactação inicial as de maior influência. O estado de compactação inicial é amplamente condicionado pelo sistema de manejo 
adotado e a intensidade do uso do solo, visto que máquinas agrícolas, equipamentos de transporte e animais, aplicam pressões diferenciadas ao solo em função do peso e do número de passadas em um determinado local.

Solos que apresentam valores elevados do índice de compressão exibem grandes mudanças na relação ar-água-matriz quando ocorre a aplicação de uma pressão, podendo assim atingir mais facilmente condições inadequadas para o crescimento das plantas. Por outro lado, valores elevados de pressão de preconsolidação implicam geralmente em condições favoráveis para o tráfego e desfavoráveis para o crescimento das raízes, devido a que são associadas a valores elevados de resistência mecânica e densidade do solo. Estes aspectos ressaltam a importância de caracterizar o processo de compressão e de se obter o índice de compressão e a pressão de preconsolidação para solos tropicais sob diferentes sistemas de manejo quando se trata de prevenir a degradação da qualidade física de terras agrícolas. Entretanto, a determinação da curva de compressão e a obtenção dos indicadores dela derivados implicam em um processo lento e associado à aquisição de equipamentos e software de custo elevado, o qual limita a realização de pesquisas nessa área de conhecimento.

Esse fato tem levado aos pesquisadores a procurarem formas alternativas de se obter os indicadores derivados da curva de compressão por meio de relações que permitam estimá-los a partir de propriedades do solo facilmente mensuráveis como a textura, umidade, densidade e resistência do solo à penetração. Na literatura internacional há abundante informação que descreve o processo de compressão de solos formados sob a influência de clima temperado, bem como modelos que relacionam o índice de compressão e a pressão de preconsolidação com os atributos intrínsecos dos solos. Entretanto, ainda há necessidade de desenvolver pesquisas que permitam uma melhor compreensão do processo de compactação e a obtenção destes indicadores para solos desenvolvidos sob clima tropical. Nesse contexto, este trabalho testou a hipótese de que o processo de compactação e os indicadores derivados da curva de compressão de solos tropicais são influenciados diferenciadamente pelo sistema de manejo aplicado, devido às alterações que o mesmo induz nas propriedades físicas do solo. O objetivo geral deste estudo foi avaliar quantitativamente o processo de compactação e os 
indicadores "índice de compressão" e "pressão de preconsolidação" de um Latossolo Vermelho Amarelo e de um Argissolo Vermelho submetidos a diferentes sistemas e intensidade de uso e obter, se possível relações que permitam estimar esses indicadores a partir de propriedades do solo facilmente mensuráveis.

Os objetivos específicos foram:

i) determinar a influência de diferentes intensidades de tráfego no processo de compactação e nos parâmetros índice de compressão e pressão de preconsolidação de um Latossolo Vermelho Amarelo sob pomar de laranja e, a partir desses indicadores, avaliar a heterogeneidade da compactação;

ii) avaliar, a partir da quantificação da pressão de preconsolidação e o entendimento das curvas de compressão uniaxial a compressibilidade de um Argissolo Vermelho sob os sistemas de pastejo rotacionado intensivo irrigado e não irrigado;

iii) obter funções que permitam estimar a capacidade de suporte de carga do solo a partir de uma propriedade facilmente mensurável e de extrema importância para o crescimento de plantas: a resistência do solo à penetração. 


\section{REVISÃO DE LITERATURA}

Diversos fatores podem condicionar o incremento da produtividade agrícola, sendo o tipo de clima, cultura e solo, os mais indicados na literatura cientifica. Novas estratégias têm sido desenvolvidas na obtenção de incrementos da produtividade agrícola associados à manutenção da qualidade do solo. O interesse em avaliar a qualidade física do solo tem sido incrementado por considerá-lo como um componente fundamental na manutenção e ou sustentabilidade dos sistemas de produção agrícola. Apesar de vários fatores dificultarem em termos quantitativos sua definição, tem sido indicada, de uma maneira geral, como a capacidade do solo em promover ao sistema radicular condições físicas adequadas ao crescimento e à produtividade das culturas (Wu et al., 2003; Karlen et al., 2003).

A adoção de técnicas como a adubação, a irrigação e de diferentes práticas de manejo têm sido utilizadas no intuito de se obter incremento da produtividade. Entretanto, estes fatores podem originar uma série de alterações físicas ao solo. Diversos tipos de solos vêm perdendo a sua qualidade física decorrente dos processos de compactação a que estão sendo expostos. A compactação do solo, tendo atingido aproximadamente 68 milhões de hectares destaca-se a nível mundial como um dos fatores que tem limitado a qualidade física de terras agrícolas e à obtenção de maiores índices de produtividade (Soane and Van Ouwerkerk, 1994; Alakukku et al., 2003).

Para um adequado crescimento, as plantas necessitam de uma estrutura que possibilite uma área de contato entre as raízes e o solo, que assegure a obtenção de água e nutrientes e um suficiente espaço poroso para o fornecimento adequado de oxigênio. A compactação, ao causar modificações na estrutura do solo pode limitar a adsorção e a absorção de nutrientes, a infiltração e a distribuição de água e, por sua vez 
resultar em problemas no estabelecimento e no crescimento de raízes. Esta limitação é originada por alterações em atributos físicos do solo, como aumento da densidade e da resistência à penetração, da diminuição da porosidade e de modificações na continuidade dos poros (Greacen \& Sands, 1980; Lhotský et al., 1991; Flowers \& Lal, 1998).

Fatores externos e internos condicionam a resposta do solo à compactação e decorrente disso, o grau de degradação da qualidade estrutural. Os fatores externos são caracterizados, pelo tipo, pela intensidade e pela freqüência da pressão exercida seja por máquinas agrícolas, equipamentos de transporte ou pisoteio de animais e, os internos, pelas propriedades físicas, mais especificamente, pelo teor de carbono orgânico, pela textura e pela umidade do solo (Raghavan et al., 1990; Snakin et al., 1996; Assouline et al., 1997; Defossez \& Richards, 2002).

A pressão exercida sobre o solo, caracterizada como um fator externo e sendo expressa pela relação existente entre uma determinada força e uma área de contato, é dependente das especificações do pneu utilizado nas operações agrícolas, como o tipo, largura e pressão de inflação de ar. Além disso, a velocidade associada a intensidade e ou a freqüência da passagem de máquinas sobre uma área agrícola, também exercem influência no processo de compactação do solo. Em sistemas de pastejo, a intensidade do pisoteio e ou o tempo de permanência dos animais na área também determinam o grau de degradação estrutural que pode ocorrer ao solo.

Há indicações na literatura cientifica de que tratores agrícolas podem exercer ao solo um intervalo de pressão de 70 a $350 \mathrm{kPa}$ (Soane, 1986). Por outro lado, em sistemas de pastejo, o gado pode aplicar pressões que variam de 350 a $400 \mathrm{kPa}$ (Proffit et al., 1993; Betterige et al.,1999).

O teor de matéria orgânica, caracterizado como um dos fatores internos parece ser dependente da umidade do solo no momento da aplicação de determinada pressão externa. Ainda é necessário determinar a influência do teor de carbono na susceptibilidade do solo à compactação para diferentes tipos de solos e umidade (Zhang et al., 1997).

A textura apresenta grande importância no processo de compactação do solo. Quando determinada pressão externa é aplicada ao solo, o incremento da pressão 
exercida por máquinas, equipamentos de transporte ou animais faz com que ocorra um novo acomodamento e ou arranjamento das partículas, diminuindo o espaço aéreo do solo, incrementando a densidade e a resistência à penetração e, decorrente disso, aumentando a compactação (Soane, 1986; Horn \& Lebert, 1994).

Além da textura, a umidade é o fator que determina a magnitude de deformação que pode ocorrer no solo no momento da aplicação da pressão, pois atua como lubrificante entre as partículas, permitindo o deslocamento das mesmas, o qual é favorecido à medida que a umidade aumenta (Larson \& Gupta, 1980; Lerink, 1990; Smith et al., 1997; Silva et al., 2000).

Em regiões que apresentam períodos de estiagens prolongadas, o uso da irrigação tem sido indicada como uma alternativa para evitar quedas de produção agrícola. No entanto, um monitoramento inadequado desta técnica, poderá incrementar o processo de degradação da qualidade estrutural considerando que, os maiores efeitos da compactação são evidenciados principalmente quando o solo encontra-se com umidade elevada (Betterige et al., 1999).

Devido a grande variedade e interação de fatores envolvidos e, visto a grande complexidade do tema, pesquisas sobre compressibilidade têm surgido no intuito de avaliar detalhadamente o processo de compressão quando determinada pressão externa é exercida ao solo.

A compressibilidade é um termo utilizado desde 1933 na área de engenharia civil (Soane, 1990), e caracterizada pela facilidade com que o solo diminui de volume quando submetido à determinada pressão (Horn \& Lebert, 1994). A compressão refere-se ao processo que descreve o decréscimo de volume do solo quando alguma pressão externa é aplicada. Este termo compreende duas definições amplamente utilizadas: i) a consolidação e ii) a compactação do solo. A consolidação refere-se a compressão de solos saturados enquanto que a compactação refere-se a compressão de solos não saturados (Gupta \& Allmaras, 1987; Koolen, 1992).

O entendimento do processo de compressão dos solos envolve a avaliação das curvas de compressão uniaxial. Esta curva é representada graficamente pela relação 
entre algum parâmetro relacionado com a estrutura do solo, mais freqüentemente, o índice de vazios ou a densidade com o logaritmo da pressão aplicada.

A partir da construção da curva, duas regiões distintas podem ser observadas, a reta de compressão virgem, porção linear onde são evidenciadas as primeiras pressões exercidas ao solo, cuja aplicação de qualquer nível de pressão condicionará deformações plásticas e irrecuperáveis e, a curva de compressão secundária que representa os níveis de pressões experimentadas pelo solo no passado. Esta parte da curva apresenta forma curvilinear, onde ocorrem deformações pequenas, elásticas e recuperáveis, sendo a região da curva em que o solo deve ser cultivado ou trafegado, sem que ocorra a compactação adicional (Lebert \& Horn, 1991; Dias Júnior \& Pierce, 1996).

Indicadores da qualidade física, relacionados à compressibilidade e, obtidos a partir da curva de compressão uniaxial têm sido reconhecidos e ampliados para estabelecer condições adequadas ao desenvolvimento das plantas (Gupta \& Allmaras, 1987; Earl, 1997; Silva et al., 2002; Mosaddeghi et al, 2003).

O índice de compressão, determinado pela inclinação da reta de compressão virgem é um indicativo da susceptibilidade do solo à compactação (Larson et al., 1980; Silva et al., 2000). Elevados valores do índice de compressão condicionam grandes mudanças na relação ar-água-matriz quando determinada pressão externa é exercida ao solo, proporcionando normalmente condições desfavoráveis ao crescimento das plantas (Gupta \& Allmaras, 1987).

A pressão de preconsolidação é uma estimativa da capacidade de suporte de carga do solo (Lebert \& Horn, 1991; Dias Júnior \& Pierce, 1996). Deformações elásticas e reversíveis são observadas quando a pressão aplicada ao solo for menor que a pressão de preconsolidação. Quando a força exceder a este valor, as deformações serão irreversíveis e plásticas (Horn \& Lebert, 1994). A pressão de preconsolidação deve ser a pressão máxima aplicada ao solo para que a compactação adicional seja evitada. Reflete a história de tensão do solo, indicando a maior pressão que o solo já suportou no passado (McNabb \& Boersma, 1996; Assouline et al. 1997; Oliveira et al., 2003). Valores elevados de pressão de preconsolidação implicam geralmente em condições favoráveis para o tráfego e inadequadas para o crescimento das raízes, devido a que são associadas 
a valores elevados de resistência mecânica e densidade do solo (Dias Júnior \& Pierce, 1996; Kondo \& Dias Júnior, 1999).

Ambos os indicadores são influenciados pelas propriedades físico-mecânicas do solo, sendo a umidade, a textura e o estado de compactação inicial, fatores de maior importância no estudo do processo de compressão.

A compactação inicial é amplamente condicionado pelo sistema de manejo adotado. No entanto, a maioria dos estudos de compressibilidade têm dado ênfase a reta de compressão virgem, a qual define deformações irrecuperáveis, não levando em consideração a história de tensão do solo causada por diferentes tipos de manejo (Dias Júnior \& Pierce, 1996).

A primeira abordagem sobre os parâmetros de compressibilidade foi desenvolvida a partir de Casagrande (1936), que indicou a importância e os procedimentos utilizados na obtenção destes indicadores.

Posteriormente, estudos sobre a reta de compressão virgem à diferentes teores de umidade e solos foram desenvolvidos por Larson et al. (1980). Os autores apresentaram as curvas de compressão uniaxial e avaliaram a susceptibilidade à compactação de oito tipos de solos com ampla variação de umidade e de argila. Foi demonstrado um modelo linear que permite estimar a densidade final ou o grau de degradação estrutural do solo a partir do conhecimento da pressão aplicada. Todas as curvas de compressão uniaxial foram condicionadas pelo grau de compactação inicial e pelo teor de umidade do solo, corroborando as observações de Larson \& Gupta (1980).

A modelagem tem apresentado grande potencialidade para o entendimento do processo de compressão dos solos. Internacionalmente, McNabb \& Boersma (1993) desenvolveram um modelo que revela com precisão a estimativa da densidade do solo em todo o intervalo de pressão aplicada, sugerindo que este modelo possa ser utilizado para estimar o grau de degradação ou a densidade final a ser atingida quando uma determinada pressão é exercida. O modelo é de grande validade, uma vez que a densidade final pode ser estimada a partir do conhecimento da densidade inicial e das pressões a que o solo será submetido. A inclusão dos valores da pressão de 
preconsolidação no modelo, possibilita determinar o valor de densidade que poderá causar a compactação adicional ao solo.

Dias Júnior \& Pierce (1995) desenvolveram um modelo não linear que permite determinar a pressão máxima que o solo pode suportar sem sofrer compactação adicional baseando-se nos diferentes teores de umidade de solos de regiões temperadas. No Brasil, Kondo (1998) utilizando o mesmo modelo, em Latossolos da região de Lavras demonstrou que a compressibilidade do solo é influenciada por diferentes sistemas de manejo.

Kondo \& Dias Júnior (1999) avaliaram a pressão de preconsolidação em Latossolos sob diferentes teores de umidade nas condições de cultura anual, mata e pastagem. As curvas de compressão uniaxial refletiram o efeito do manejo e da umidade na capacidade de suporte de carga do solo. Os autores, sugeriram que a pressão de preconsolidação seja considerada como um indicador quantitativo da sustentabilidade estrutural (Dias Junior \& Pierce, 1996).

Silva et al. (2000) em dois tipos de solos de importância agrícola no RS, submetidos a diferentes sistemas de manejo testaram modelos para estimar a pressão de preconsolidação e o índice de compressão utilizando valores de densidade inicial a diferentes graus de saturação. Os autores concluíram que existe dependência entre o grau de saturação, sistemas de manejo e a compressibilidade do solo, corroborando os resultados de Kondo \& Dias Júnior (1999).

Salire et al. (1994) apresentaram uma relação linear e positiva entre a pressão de preconsolidação e a densidade do solo. Menores valores de densidade estiveram associados à menor capacidade de suporte de carga do solo. Os autores concluíram ainda que a pressão de preconsolidação pode ser usada como um indicador seguro na estimativa da pressão máxima a ser exercida com vistas à prevenção da compactação do solo.

Imhoff et al. (2004) estudando vários tipos de solos com ampla variação de textura sob cultivo de cana de açúcar, em uma toposseqüência concluíram que a compressibilidade é dependente de diversos atributos físicos, especificamente do estado de compactação inicial do solo. Os autores apresentaram um modelo que incorpora o 
efeito da umidade, matéria orgânica, textura e densidade do solo na pressão de preconsolidação.

Indicações na literatura científica em geral, ressaltam a importância de caracterizar o processo de compressão e de se obter o índice de compressão e a pressão de preconsolidação quando se trata de prevenir a degradação da qualidade física dos solos. Em sistemas mecanizados, o conhecimento dos valores do índice de vazios, ou de densidade e dos indicadores resultantes da curva de compressão uniaxial a cada pressão exercida, pode apresentar grande relevância na adequação futura de máquinas que exerçam menores pressões sobre o solo e na redução do impacto causado a qualidade estrutural de terras agrícolas (Kondo \& Dias Júnior, 1999; Mosaddeghi et al., 2003).

Portanto, apesar da reconhecida importância do entendimento deste tema, a determinação da curva de compressão e a obtenção dos indicadores dela derivados têm limitado a realização de pesquisas nesta área de conhecimento. Há necessidade de desenvolver relações básicas e pesquisas aplicadas que permitam um melhor entendimento do processo de compressão dos solos de regiões tropicais.

A quantificação da pressão de preconsolidação, mais especificamente é um processo lento e está associada à aquisição de equipamentos de custo elevado. Os métodos existentes de determinação deste parâmetro exigem a construção da curva de compressão uniaxial que baseia-se na aplicação de pressões sucessivas a intervalos regulares de tempo.

O método padrão de determinação gráfica da pressão de preconsolidação proposto por Casagrande (1936) é normalmente utilizado em estudos de compressibilidade do solo. Este método fundamenta-se em encontrar um ponto de mínima curvatura da curva de compressão e por este ponto é traçada uma reta tangente e outra paralela ao eixo das abscissas, determinando-se após a linha bissetriz do ângulo formado entre estas duas retas. Posteriormente, é identificado, o ponto de intersecção entre a linha bissetriz e o prolongamento da reta de compressão virgem, o qual é indicado no eixo das abscissas como a pressão de preconsolidação.

O emprego de técnicas de regressão (Lebert \& Horn, 1991; McBride \& Joosse, 1996; Assouline et al., 1997; Fritton, 2001; Assouline, 2002) e, de softwares com 
programas específicos também auxiliam a determinação da pressão de preconsolidação do solo. Dias Júnior \& Pierce (1995) desenvolveram um modelo computacional que associa diferentes metodologias para a avaliação deste parâmetro. Da mesma forma, Veenhof \& McBride (1996) testaram diversos modelos para a determinação deste indicador.

Mais recentemente, Imhoff et al. (2004) utilizaram um programa desenvolvido dentro do software Mathcad (Mathsoft Inc., 2000) para a determinação da pressão de preconsolidação. Na primeira etapa, o programa calcula o ponto de mínima curvatura da curva de compressão uniaxial. Para isso, é calculada a segunda derivada de cada ponto da curva, sendo o ponto de mínima curvatura, aquele em que a segunda derivada atinge o valor mínimo. Em etapas sucessivas, a quantificação da pressão de preconsolidação é feita utilizando-se o método padrão gráfico proposto por Casagrande (1936). Uma vantagem da utilização deste software é que exclui a subjetividade que o método manual oferece na determinação da pressão de preconsolidação.

Visto a reconhecida importância do conhecimento da pressão de preconsolidação em sistemas agrícolas, pesquisadores têm procurado formas alternativas de se obter este indicador por meio de relações que permitam estimá-la a partir de propriedades facilmente mensuráveis em solos de regiões tropicais.

A pressão de preconsolidação é uma estimativa da resistência do solo na qual a elongação das raízes cessa, ou seja, solos com valores elevados de pressão de preconsolidação apresentam maior probabilidade de reduzir o crescimento das raízes (Canarache et al., 2000; Mosaddeghi et al., 2003). Baseado nesta indicação, a resistência do solo à penetração, um dos atributos diretamente relacionados com a produtividade das culturas e, amplamente utilizada para estabelecer condições restritivas ao desenvolvimento radicular e à produtividade das culturas apresenta grande validade para prever a capacidade de suporte de solos parcialmente saturados (Veen \& Boone, 1990; Boone et al., 1994; Canarache et al., 2000; Kirby \& Bengough, 2002; Mosaddeghi et al., 2003).

Uma estimativa da pressão de preconsolidação, obtida a partir da quantificação da resistência do solo à penetração compreende uma medida útil do estado mecânico do 
solo para o seu uso, manejo e planejamento de sistemas de mecanização agrícola. Além disso, a avaliação da resistência à penetração apresenta como vantagem de ser um processo rápido e requerer equipamentos de baixo custo (Lipiec \& Hatano, 2003; Nevens \& Reheul et al., 2003).

Culley \& Larson (1987) avaliando a compressibilidade de um solo franco argiloso sob cultivo de milho e submetidos a dois diferentes sistemas de manejo, apresentaram uma relação linear entre a pressão de preconsolidação e a resistência do solo à penetração. Os autores concluíram que a resistência à penetração é um parâmetro útil para caracterizar a capacidade de suporte de carga de um solo em condições de campo. Os resultados fornecem forte evidência da utilidade da resistência à penetração na estimativa da pressão de preconsolidação do solo.

Posteriormente, Burguer et al. (1988) numa estimativa da compressibilidade mostraram que a variação da capacidade de suporte de carga com a profundidade do solo é comparável com a variação da resistência à penetração.

Pesquisas têm mencionado a relação existente entre a resistência do solo à penetração e a pressão de preconsolidação. Farrel \& Greacen (1966) estudaram a compressibilidade de solos de textura franco à argilosa à dois níveis de umidade. Canarache et al. (2000) avaliaram a compressibilidade em um experimento de campo à longo prazo, em solos franco siltosos e argilo siltosos. Os autores indicaram uma relação de 10:1 entre estes dois parâmetros. Recentemente, esta relação foi confirmada por Mosaddeghi et al. (2003) para solos franco argilo siltosos de regiões de clima temperado.

Considerando-se esta relação e que limitações ao crescimento radicular ocorrem normalmente a valores de resistência do solo à penetração superiores a $2 \mathrm{MPa}$, o estabelecimento de uma estimativa da capacidade de suporte de carga à valores de resistência à penetração considerados não impeditivos ao crescimento de raízes, poderá evitar a degradação da qualidade estrutural de solos submetidos a diferentes sistemas de manejo (Taylor et al., 1966).

Apesar de várias pesquisas abordarem a importância do conhecimento da compressibilidade para diferentes tipos de solos e de sistemas produtivos das regiões de 
clima temperado (Lebert \& Horn, 1991; Dias Júnior \& Pierce, 1995; Veenhof \& McBride, 1996) e tropical (Silva et al., 2000; Silva et al., 2002), há um consenso generalizado sobre a necessidade de ampliar as pesquisas nesta área de conhecimento que possibilitem uma melhor compreensão do processo de compactação e dos indicadores derivados da curva de compressão de solos desenvolvidos em regiões de clima tropical e submetidos a diferentes sistemas de manejo. 


\section{HETEROGENEIDADE DA COMPACTAÇÃO DE UM LATOSSOLO VERMELHO AMARELO SOB POMAR DE LARANJA}

\section{Resumo}

A heterogeneidade na compactação do solo, induzida por diferentes intensidades de tráfego, pode limitar a produção em áreas citrícolas. O objetivo desse trabalho foi quantificar as curvas de compressão e os parâmetros índice de compressão (IC) e pressão de preconsolidação $\left(\sigma_{\mathrm{p}}\right)$, em diferentes posições de amostragem, para avaliar o processo e a heterogeneidade da compactação induzidos pelo tráfego de máquinas em um Latossolo Vermelho Amarelo sob pomar de laranja. A amostragem foi realizada em 40 pontos distribuídos aleatoriamente (10 em cada posição). Após saturadas com água e equilibradas no potencial $(\psi)$ : $-10 \mathrm{kPa}$, as amostras foram pesadas e submetidas ao ensaio de compressão uniaxial com a aplicação sucessiva e contínua de pressões de 25, 50, 100, 200, 400, 600, 800, 1000, 1300 e $1600 \mathrm{kPa}$. Os resultados indicaram que o processo de compactação foi influenciado pela intensidade de tráfego, sendo os valores de $\sigma_{\mathrm{p}}$ significativamente superiores nas posições de amostragem entre rodado, rodado e projeção da copa em relação à linha de cultivo e os valores de IC similares em todas as posições de amostragem. A $\sigma_{\mathrm{p}}$ foi o indicador mais sensível na avaliação da heterogeneidade da compactação induzida pelo tráfego de máquinas em áreas citrícolas. Palavras-chave: pomar de laranja, índice de compressão, pressão de preconsolidação, posição de amostragem. 


\section{COMPACTION HETEROGENEITY OF AN HAPLUSTOX UNDER AN ORANGE ORCHARD}

\section{Summary}

The soil compaction heterogeneity, induced by different traffic intensities, may reduce the productivity of citrus trees. The objective of this research was to quantify the compression curves, and the compression index (CI) and the preconsolidation pressure $\left(\sigma_{\mathrm{p}}\right)$ in different sampling positions to evaluate the compaction process and heterogeneity, induced by the machinery traffic, in an Haplustox under an orange orchard. The sampling was carried out in forty sites randomly distributed (ten samples in each position). The samples were saturated in water and equilibrated in the matric potential $(\psi)$ : $-10 \mathrm{kPa}$. Then, they were weighted and submitted to a uniaxial compression test, being applied the following pressures: 25, 50, 100, 200, 400, 600, 800, 1000, 1300 and $1600 \mathrm{kPa}$. The results indicated that the compaction process was influenced by the traffic intensity, being $\sigma_{\mathrm{p}}$ values significantly higher in the between wheel track, wheel track, and canopy projection positions than in the row position, and the CI values were similar in all positions. The $\sigma_{\mathrm{p}}$ was the most sensitive indicator to evaluate the compaction heterogeneity induced by the traffic of agricultural machinery in areas cultivated with citrus trees.

Key words: orange orchard, compression index, preconsolidation pressure, sampling position.

\subsection{Introdução}

O Brasil com mais de um milhão de hectares de plantas cítricas tornou-se, na década de 80, o maior produtor mundial de laranjas. O Estado de São Paulo é responsável por $70 \%$ da produção nacional de laranjas e por $98 \%$ da produção de suco concentrado (ABECitrus, 2004).

A produtividade em áreas citrícolas é dependente da interação entre os fatores que constituem o ecossistema do pomar, como o clima e o solo, que determinam a 
produção e a qualidade dos frutos (Moreno \& Gascon, 1994). A compactação do solo é um dos fatores limitantes na produção de citrus (Oliveira \& Merwin, 2001) e é condicionada por fatores externos, especificamente, pelo tipo, intensidade e frequência da pressão aplicada por máquinas agrícolas (Soane et al., 1981; Lerink, 1990; Lebert \& Horn, 1991) e por fatores internos, ou seja, a textura, a estrutura, ciclos de secagem e umedecimento e densidade do solo (Culley \& Larson, 1987; Horn, 1988).

A intensidade do tráfego de máquinas condiciona a resposta do solo à compressão, ou seja, determina o grau de alteração das propriedades físicas (Soane et al., 1982; Culley \& Larson, 1987; Gysi, 2001). Em um ano agrícola, a freqüência comum de quinze passadas de trator para a realização de operações, como adubações e pulverizações, pode intensificar ainda mais a degradação da estrutura do solo em áreas citrícolas (Stolf, 1987; Tersi \& Rosa, 1995).

Os efeitos dos sistemas de preparo sobre as propriedades físicas do solo podem ser manifestados diferentemente em relação à linha de cultivo. Sanches et al. (1999) avaliando o impacto do cultivo de citrus em propriedades físicas observaram maiores valores de densidade do solo na entrelinha. Em função da carência de pesquisas que abordem este tema, existe a necessidade de estudos que avaliem o processo e a heterogeneidade da compactação induzidos por diferentes intensidades de tráfego de máquinas em áreas citrícolas.

A quantificação da compactação nestas áreas pode ser incrementada a partir da utilização de parâmetros de compressão do solo, obtidos por meio de ensaios de compressão uniaxial, como o índice de compressão (IC) e a pressão de preconsolidação $\left(\sigma_{\mathrm{p}}\right)$. Esses parâmetros, além de auxiliarem na análise do processo de compactação de solos agrícolas (Bailey et al., 1986; Gupta \& Allmaras, 1987; Fritton, 2001), têm sido utilizados na adequação de máquinas que exerçam menores pressões sobre o solo (Pytka \& Dabrowski, 2001).

Este trabalho testou a hipótese de que o processo de compactação depende da intensidade do tráfego em áreas citrícolas e que os parâmetros de compressão do solo podem ser utilizados para avaliar o processo e a heterogeneidade da compactação nestas áreas. Nesse sentido, objetivou-se quantificar as curvas de compressão e os parâmetros 
índice de compressão (IC) e pressão de preconsolidação $\left(\sigma_{\mathrm{p}}\right)$, em diferentes posições de amostragem, para avaliar o processo e a heterogeneidade da compactação induzidos pelo tráfego de máquinas em um Latossolo Vermelho Amarelo sob pomar de laranja.

\subsection{Material e Métodos}

O estudo foi conduzido em um pomar de laranja pêra (Citrus sinensis (L.) Osbeck) sobre o porta-enxerto limão “cravo” (Citrus limonia (L.) Osbeck), implantado no mês de março do ano de 1991, pertencente ao Departamento de Produção Vegetal (Setor de Horticultura) da ESALQ/USP, localizado na cidade de Piracicaba, SP. A área total do pomar é de $24.128 \mathrm{~m}^{2}$ implantado com espaçamento de 8 x $4 \mathrm{~m}$. O solo é classificado como um Latossolo Vermelho Amarelo distrófico (Embrapa, 1999) de textura franco arenosa (80 $\mathrm{g} \mathrm{kg}^{-1}$ de silte, $750 \mathrm{~g} \mathrm{~kg}^{-1}$ de areia e $170 \mathrm{~g} \mathrm{~kg}^{-1}$ de argila).

O controle de ervas daninhas na linha do cultivo é feito três vezes ao ano com a aplicação de herbicida e roçagem nas entrelinhas utilizando uma roçadeira Baldan RP 1700, com peso aproximado de $358 \mathrm{~kg}$. O controle de ácaros, mosca das frutas e bicho furão é feito duas vezes ao ano, utilizando um pulverizador de aproximadamente $825 \mathrm{~kg}$ e capacidade para 2000 litros. Em todas as operações agrícolas foi utilizado um trator MF 275 (4 x 2) com um peso aproximado de $3900 \mathrm{~kg}$ e pneus dianteiros do tipo 7.5 16F2 cuja pressão de inflação de ar dos pneus é de 240 kPa e traseiros do tipo 18.4 30R1, que utilizam uma pressão de 96,5 kPa.

A amostragem foi realizada no mês de agosto do ano de 2001 distribuindo-se aleatoriamente dez pontos no entre rodado (ER), dez pontos no rodado (R), dez pontos na projeção da copa (PC) e dez pontos na linha de cultivo (L) (Figura 1). Em cada ponto foi retirada, na camada superficial, uma amostra indeformada com anel volumétrico de 7 cm de diâmetro interno e 2,5 cm de altura, totalizando 40 amostras.

As amostras foram saturadas com água por 48 horas e submetidas ao potencial (భ): - 10 kPa, em câmaras de Richards (Klute, 1986). Após o equilíbrio, cada amostra foi pesada e submetida ao ensaio de compressão uniaxial. Este ensaio consistiu na aplicação sucessiva e contínua de pressões crescentes e preestabelecidas de 25, 50, 100, 
200, 400, 600, 800, 1000, 1300 e $1600 \mathrm{kPa}$, por cinco minutos, período suficiente para atingir 99\% da deformação máxima (Silva et al., 2002).

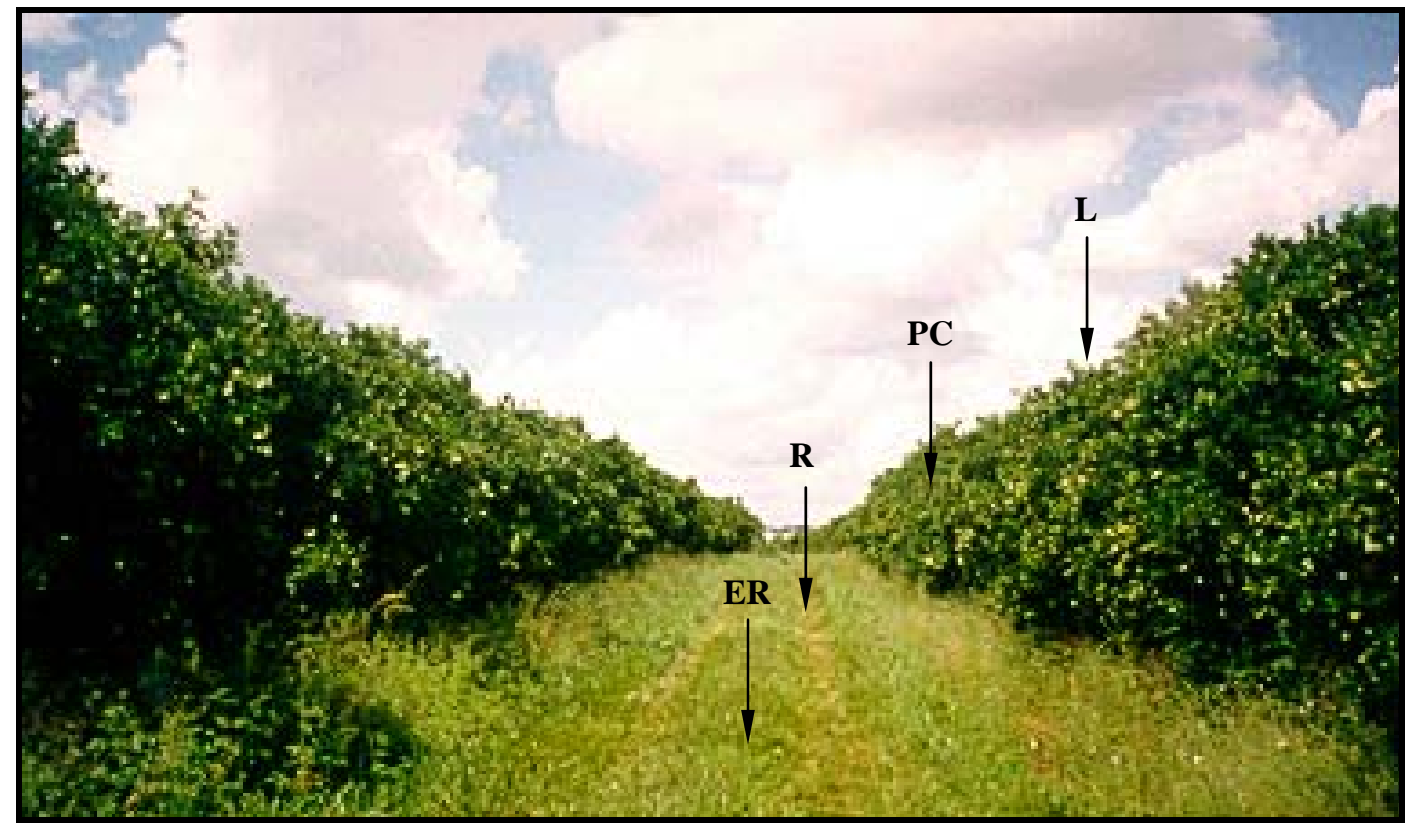

Figura 1 - Posições de amostragem: ER (entre rodado); R (rodado), PC (projeção da copa) e L (linha de cultivo) no pomar de laranja

O deslocamento vertical a cada pressão aplicada foi registrado por meio de um sensor ligado a um sistema de aquisição de dados automatizado MCT 2000 - Satron (MIRAE Engineering, Inc., Buscan Korea) (Figura 2).

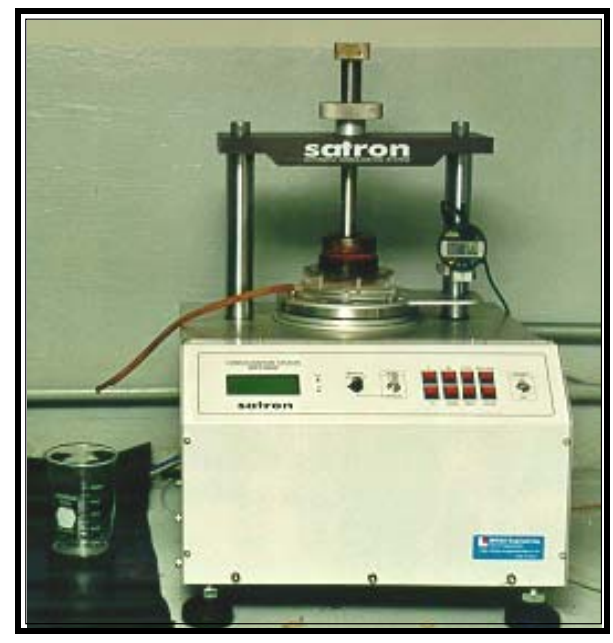

Figura 2 - Equipamento utilizado para a realização dos testes de compressão uniaxial 
Posteriormente, as amostras foram secadas em estufa a $105^{\circ} \mathrm{C}$, por 24 horas e a partir destes valores foi determinada para cada amostra a densidade do solo, conforme Blake \& Hartge (1986a).

O ensaio permitiu a obtenção da curva de compressão uniaxial do solo, que relaciona o logaritmo da pressão aplicada (eixo x) versus a densidade do solo (eixo y). A partir destas curvas, foram obtidos os valores de índice de compressão (IC) e de pressão de preconsolidação $\left(\sigma_{\mathrm{p}}\right)$, utilizando o método de Casagrande (1936) com auxílio do software Mathcad (Mathsoft Inc., 2000) conforme Imhoff et al. (2004).

Os resultados foram submetidos a análise de variância, sendo as médias nas diferentes posições de amostragem comparadas utilizando o teste que considera a diferença mínima significativa a 5\%, utilizando o software SAS (Statistical Analysis System Institute, 1991).

\subsection{Resultados e Discussão}

As curvas de compressão uniaxial, para cada posição de amostragem (média de 10 repetições), no potencial $(\psi)$ : - $10 \mathrm{kPa}$ são apresentadas na Figura 3. Houve um menor incremento da densidade do solo com a aplicação das pressões nas posições ER (1,54 a 1,81 $\left.\mathrm{Mg} \mathrm{m}^{-3}\right), \mathrm{R}\left(1,58\right.$ a $\left.1,83 \mathrm{Mg} \mathrm{m}^{-3}\right)$ e PC (1,58 a 1,85 $\left.\mathrm{Mg} \mathrm{m}^{-3}\right)$ em relação a L (1,41 a 1,73 $\left.\mathrm{Mg} \mathrm{m}^{-3}\right)$. Este comportamento pode estar associado ao maior estado de compactação inicial observado nas posições ER, R e PC, gerado pelo tráfego de máquinas no pomar para executar as operações agrícolas de adubação, pulverização e controle de plantas daninhas, o que corrobora os resultados descritos por Gérik et al. (1987).

Nas curvas de compressão uniaxial são identificadas duas regiões clássicas: (i) uma região em que ocorrem deformações pequenas, elásticas e recuperáveis, chamada de curva de compressão secundária (CCS) e (ii) outra região representada pela porção onde ocorrem deformações plásticas e não recuperáveis, chamada de reta de compressão virgem (RCV). 


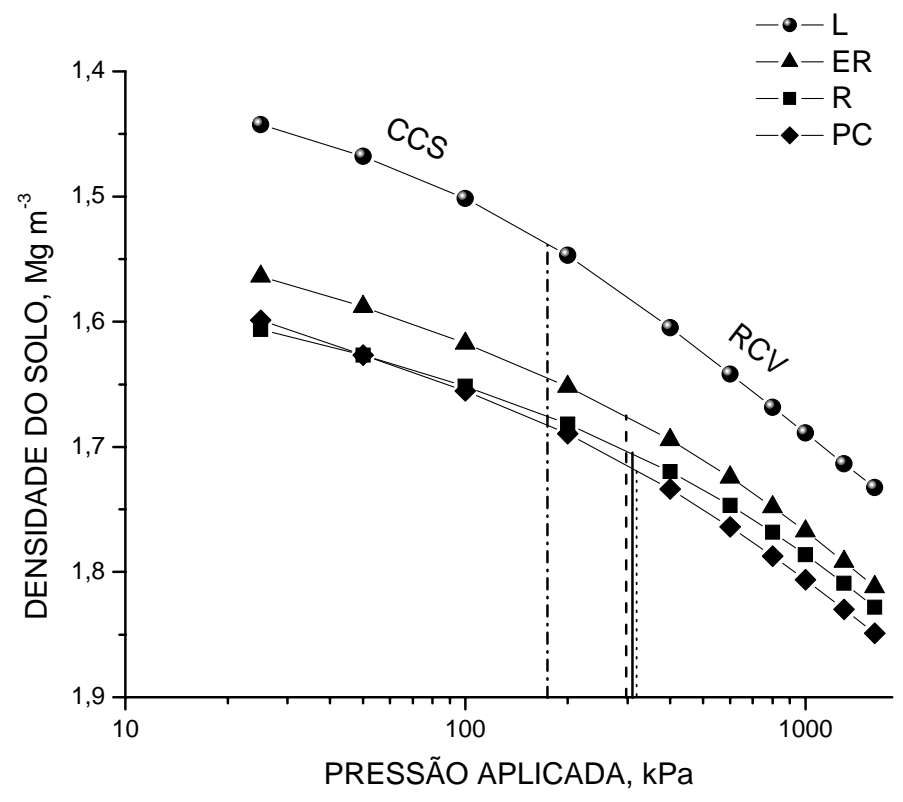

Figura 3 - Curvas de compressão uniaxial nas posições linha (L), entre rodado (ER), rodado (R) e projeção da copa (PC) no potencial ( $\psi)$ : - $10 \mathrm{kPa}$. As linhas verticais separam as duas regiões na curva de compressão uniaxial (CCS e RCV) nas posições L, ER, R e PC, respectivamente

Essas duas regiões estão mais evidentes na posição L. O solo nesta posição, provavelmente por estar menos compactado, apresentou maior deformação do que nas demais posições de amostragem (Figura 3).

$\mathrm{O}$ aumento do estado inicial de compactação, $\mathrm{PC}>\mathrm{R}>\mathrm{ER}>\mathrm{L}$, favoreceu $\mathrm{o}$ deslocamento das linhas que separam a CCS da RCV para a direita, indicando que a compactação adicional ocorrerá a valores superiores de pressões aplicadas nas posições PC, R e ER, em relação a L. O comportamento do solo à compressão nas posições PC, $\mathrm{R}$, e ER foi semelhante, como indica a proximidade das linhas que separam a CCS e a RCV. Esta semelhança pode estar relacionada a uma condição similar de compactação a que foi submetido o solo nestas posições.

A partir das duas regiões identificadas na curva de compressão (CCS e RCV) foram calculados os parâmetros índice de compressão (IC) e pressão de preconsolidação $\left(\sigma_{\mathrm{p}}\right)$. O IC, sendo o coeficiente angular da RCV, representa a susceptibilidade de solo à 
compactação e a $\sigma_{\mathrm{p}}$, sendo o limite entre as regiões onde ocorrem as deformações elásticas (CCS) e plásticas (RCV) do solo, indica a capacidade de suporte de carga do solo. Os momentos estatísticos para estes indicadores estão apresentados na Tabela 1.

Tabela 1. Momentos estatísticos dos parâmetros da curva de compressão uniaxial

\begin{tabular}{ccccccc}
\hline Variáveis & N & Média & $\begin{array}{c}\text { Desvio } \\
\text { padrão }\end{array}$ & Mínimo & Máximo & CV (\%) \\
\hline IC & 40 & 0,19 & 0,02 & 0,14 & 0,24 & 10,34 \\
$\sigma_{\mathrm{p}}$ & 40 & 274 & 94,72 & 75,00 & 547,00 & 34,54 \\
\hline
\end{tabular}

IC = índice de compressão; $\sigma_{\mathrm{p}}=$ pressão de preconsolidação $(\mathrm{kPa})$

O maior coeficiente de variação da $\sigma_{\mathrm{p}}(34,54 \%)$ em relação ao IC $(10,34 \%)$ indicou que a $\sigma_{\mathrm{p}}$ esteve associada com a heterogeneidade do estado físico do solo nas diferentes posições de amostragem e que o IC esteve associado com as propriedades intrínsecas do solo.

O valor médio do IC $(0,19)$ foi similar ao encontrado por Imhoff et al. (2004) para Latossolos de textura semelhante a deste estudo. Estes autores estudaram uma toposeqüência em Latossolos com ampla variação no teor de argila e verificaram que o IC aumentou com o teor de argila até o valor aproximado de $30 \%$, permanecendo constante a partir deste valor. Esse fato pode explicar porque Kondo \& Dias Junior (1999) e Silva et al. (2000) apresentaram valores de IC superiores aos encontrados neste estudo. Os Latossolos estudados por estes autores apresentaram teores de argila mais elevados.

O valor encontrado do IC indica que o solo estudado apresenta reduzida susceptibilidade à compactação. A textura determina o tipo, a forma e a distribuição do tamanho das partículas do solo e, como conseqüência, o número de contatos entre elas, controlando, assim, a resistência do solo à deformação (Harte, 2000). Solos com textura grossa são menos susceptíveis à compactação do que aqueles com textura fina (Horn, 1988; Horn \& Lebert, 1994). A menor susceptibilidade do solo à compactação é 
decorrente do maior atrito entre as partículas de solos arenosos, o que dificulta a movimentação destas partículas para posições de maior proximidade (deformação).

A análise de variância revelou que diferenças na intensidade do trafego de máquinas agrícolas não exerceram influência significativa sobre o IC (F = 2,39; $\mathrm{P}<$ 0,0850), confirmando o fato de que o IC é um indicador relacionado principalmente às propriedades intrínsecas do solo, como foi assinalado por Larson et al. (1980) e Horn (1988).

Por outro lado, a $\sigma_{\mathrm{p}}(\mathrm{F}=7,69 ; \mathrm{P}<0,0004)$ foi significativamente relacionada com a posição de amostragem, refletindo as condições físicas do solo, induzidas pelas diferenças na intensidade do tráfego de máquinas agrícolas. Visto que, somente este indicador apresentou-se significativamente relacionado com o local de amostragem, procedeu-se uma análise de comparação de médias, para se verificar diferenças significativas da $\sigma_{\mathrm{p}}$ nas diferentes posições de amostragem no pomar.

Não houve diferenças significativas, ao nível de 5\%, entre os valores médios de $\sigma_{\mathrm{p}}$ nas posições PC (316 kPa), R (309 kPa) e ER (298 kPa). Entretanto, esses valores foram significativamente maiores que o valor médio de $\sigma_{\mathrm{p}}$ na $\mathrm{L}(174 \mathrm{kPa})$.

Os maiores valores da $\sigma_{\mathrm{p}}$ nas posições PC, R e ER indicaram maior capacidade de suporte de carga ao tráfego de máquinas agrícolas, até que o processo de compactação adicional ocorra. O menor valor da $\sigma_{\mathrm{p}}$ na $\mathrm{L}$ evidenciou que o solo suportou menores pressões no passado, justificado pela ausência de operações mecanizadas nestas áreas.

Considerando que o valor da $\sigma_{\mathrm{p}}$ é resultado dos níveis de pressões aplicadas no passado e, também, de processos de secagem umedecimento do solo (Rajaram \& Erbach, 1998; Silva et al., 2000), os valores da $\sigma_{\mathrm{p}}$ obtidos nas posições PC, R, e ER podem ser atribuídos à pressão aplicada no solo pelo trator juntamente com os equipamentos utilizados para a realização das operações agrícolas no pomar. Estes equipamentos normalmente apresentam pneus mais estreitos do que os utilizados no trator, podendo exercer elevados valores de pressão ao solo. Febo \& Pessina (2002) afirmaram que a pressão exercida por equipamentos agrícolas são normalmente 
superiores a pressão exercida pelo trator e que tanques pulverizadores podem exercer pressões médias de aproximadamente $300 \mathrm{kPa}$, valor semelhante ao obtido neste estudo.

A $\sigma_{\mathrm{p}}$ foi mais sensível que o IC para discriminar mudanças relacionadas à heterogeneidade espacial da compactação do solo, visto que somente este parâmetro foi significativamente influenciado por diferenças na intensidade do tráfego de máquinas agrícolas no pomar.

A Figura 4 ilustra na entrelinha de cultivo um incremento da $\sigma_{\mathrm{p}}$ de 70 a $80 \%$ em relação L.

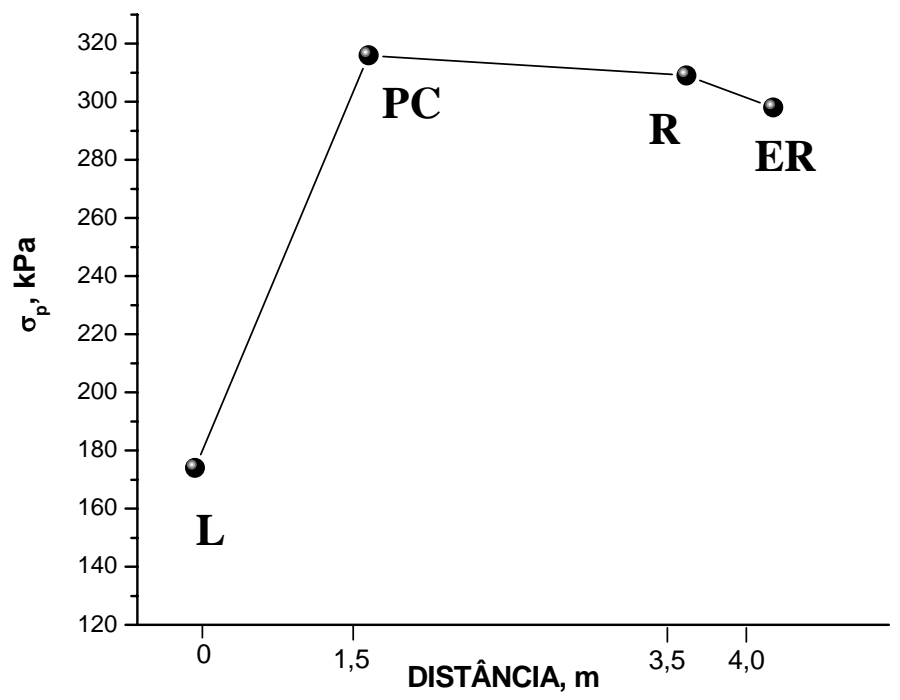

Figura 4 - Valores médios da $\sigma_{\mathrm{p}}$ nas posições L (linha), PC (projeção da copa), R (rodado) e ER (entre rodado), no potencial $(\psi)$ : $-10 \mathrm{kPa}$, distanciados a partir da L de 1,5 m (PC), 3,5 m (R) e 4,0 m (ER) na área de citrus

As posições de amostragem localizadas a partir de 1,5 m (PC, R, ER) apresentaram valores superiores de $\sigma_{p}$ e condições homogêneas de compactação, indicando a ocorrência de tráfego de máquinas agrícolas em toda a entrelinha e, por sua vez, condições menos favoráveis para o crescimento radicular. Trabalhos têm demonstrado que limitações ao crescimento radicular ocorrem a valores de resistência do solo à penetração (RP) superiores a 2 MPa (Taylor et al., 1966). Por outro lado, 
pesquisas têm demonstrado que existe uma relação entre a RP e a $\sigma_{\mathrm{p}}$, sendo RP $=10 \sigma_{\mathrm{p}}$ (Canarache et al., 2000; Mosaddeghi et al., 2003). Assumindo-se essa relação para a área estudada, e de acordo com os resultados de $\sigma_{\mathrm{p}}$ obtidos, espera-se valores de RP próximos a $3 \mathrm{MPa}$ na entrelinha de cultivo. Decorrente disto, pode ser que o crescimento das raízes das árvores nestas áreas fique restrito a $\mathrm{L}$ e a uma distância de 1,5 m em relação a L, o que pode vir a condicionar uma baixa produtividade no pomar.

Para evitar a degradação estrutural do solo, cuidados especiais devem ser implementados nestas áreas. Nesse sentido, a adoção de linhas permanentes de tráfego de máquinas agrícolas ou a implementação de técnicas de subsolagem na entrelinha do cultivo podem contribuir para a manutenção da qualidade física do solo e para o incremento da produtividade destas áreas.

\subsection{Conclusões}

Os resultados comprovam a hipótese de que o processo de compactação é influenciado pela intensidade do tráfego em áreas citrícolas, como ilustrado pelas curvas de compressão que variaram com a posição de amostragem.

Os resultados indicam que a $\sigma_{p}$ pode ser utilizada na avaliação da heterogeneidade da compactação induzida por diferentes intensidades de tráfego de máquinas agrícolas por ser um indicador mais sensível que o IC na avaliação da heterogeneidade da compactação em áreas citrícolas e que este último não deve ser utilizado com a mesma finalidade em áreas citrícolas.

A $\sigma_{\mathrm{p}}$ foi significativamente superior nas posições de amostragem entre rodado, rodado e projeção da copa em relação à linha de cultivo, enquanto os valores de IC foram similares em todas as posições de amostragem. 


\section{COMPRESSIBILIDADE DE UM ARGISSOLO VERMELHO SOB SISTEMAS DE PASTEJO ROTACIONADO INTENSIVO IRRIGADO E NÃO IRRIGADO}

\section{Resumo}

O sistema de manejo pode afetar diferenciadamente o processo de compactação do solo, determinando o nível de degradação atingido. O objetivo desse trabalho foi avaliar a influencia do pisoteio animal na pressão de preconsolidação e nas curvas de compressão uniaxial de um Argissolo Vermelho sob os sistemas de pastejo rotacionado intensivo irrigado e não irrigado. Foram coletadas 96 amostras indeformadas de solo em quatro ciclos sucessivos de pastejo implantado com capim Tanzânia (Panicum maximum Jacq.). Após saturadas com água e equilibradas no potencial ( $\psi)$ : - $10 \mathrm{kPa}$, as amostras foram pesadas e submetidas ao ensaio de compressão uniaxial com a aplicação sucessiva e contínua de pressões de 25, 50, 100, 200, 400, 600, 800, 1000, 1300 e 1600 kPa. Os resultados indicaram que houve diferença na compressibilidade do solo sob sistemas de pastejo rotacionado intensivo irrigado e não irrigado. As curvas de compressão foram deslocadas para valores superiores de densidade do solo no sistema de pastejo rotacionado intensivo irrigado. Houve aumento dos valores de pressão de preconsolidação os quais foram significativamente superiores no quarto ciclo de pastejo no sistema de pastejo rotacionado intensivo irrigado.

Palavras-chave: pisoteio animal, pressão de preconsolidação, curva de compressão uniaxial, sistema de pastejo. 


\section{COMPRESSIBILITY OF AN HAPLUDALF UNDER NOT IRRIGATED AND IRRIGATED ROTATION SHORT-DURATION GRAZING SYSTEMS}

\section{Summary}

The management system may affect differently the soil compaction process, being one of the responsible factors for the soil degradation. The objective of this research was to evaluate the influence of the animal trampling on the preconsolidation pressure, and on the compression curves of an Hapludalf under not irrigated and irrigated rotation short-duration grazing systems. Ninety-six undisturbed cores were taken in four successive grazing cycles of Tanzania grass (Panicum maximum Jacq.). The cores were saturated in water and equilibrated in the matric potential $(\psi):-10 \mathrm{kPa}$. Then, they were weighted and submitted to an uniaxial compression test, being applied the following pressures: 25, 50, 100, 200, 400, 600, 800, 1000, 1300, $1600 \mathrm{kPa}$. The results indicated that there were differences in the soil compressibility under the not irrigated and irrigated rotation short-duration grazing systems. The soil compression curves were displaced to higher soil bulk density values in the irrigated rotation shortduration grazing system. There was an increase on the preconsolidation pressure values that were significantly higher in the fourth cycle in the irrigated rotation short-duration grazing system.

Key words: animal trampling, preconsolidation pressure, uniaxial compression curve, grazing system.

\subsection{Introdução}

O Brasil abrange uma área total de pastagens na ordem de 180 milhões de hectares, o que corresponde a mais de 20\% do território brasileiro (Zimmer et al., 2002). Um dos grandes entraves na obtenção de elevadas produtividades tem sido a compactação do solo. A compressibilidade do solo, que indica a resistência do solo ao decréscimo de volume quando submetido a uma certa pressão (Horn \& Lebert, 1994), é determinada pelas características do solo como a textura e a umidade bem como pela 
intensidade, freqüência e tipo de sistema de pastejo adotado (Stephenson \& Veigel, 1987; Defossez \& Richard, 2002).

Na literatura há indicações que os animais podem exercer pressões no solo da ordem de 350 a 400 kPa (Proffit et al., 1993; Betterige et al., 1999). Nie et al. (2001) indicaram ainda que a pressão exercida pelos animais em movimento pode ser o dobro da exercida quando eles estão parados.

Diversas técnicas têm sido utilizadas no intuito de se obter incremento da produção das pastagens, como técnicas de adubação, a adoção de diferentes sistemas de pastejo e, ultimamente, a irrigação (Chanasyk \& Naeth, 1995). No entanto, a irrigação em pastagens pode facilitar a compactação do solo, uma vez que os maiores efeitos do pisoteio de animais são evidenciados principalmente quando o solo encontra-se com umidade elevada (Tanner \& Mamaril, 1959; Betterige et al., 1999).

A densidade, a resistência do solo à penetração e a porosidade são alguns dos indicadores utilizados na avaliação da compactação do solo em áreas sob pastagens (Ferrero, 1991; Profitt et al., 1993; Silva et al., 2003). Esses indicadores caracterizam-se por avaliar o estado de compactação do solo, mas não avaliam o processo de compressão.

O entendimento do comportamento compressivo do solo baseia-se na avaliação das curvas de compressão uniaxial. A pressão de preconsolidação do solo, parâmetro obtido da curva de compressão, tem sido amplamente aceita como um indicativo de sua capacidade de suporte de carga, uma vez que a aplicação de pressões maiores a este valor podem induzir compactação adicional ao solo (Dias Júnior \& Pierce, 1996; Kondo \& Dias Júnior, 1999).

No Brasil há carência de estudos que descrevam o comportamento compressivo de solos irrigados, cultivados com pastagens e submetidos a utilização intensiva. A hipótese testada neste estudo é de que há diferença na compressibilidade de solos sob sistemas de pastejo rotacionado intensivo irrigado e não irrigado. Nesse sentido, o objetivo desse trabalho foi determinar a influência do pisoteio animal na pressão de preconsolidação e nas curvas de compressão uniaxial de um Argissolo Vermelho sob os sistemas de pastejo rotacionado intensivo irrigado e não irrigado. 


\subsection{Material e Métodos}

O estudo foi conduzido em uma área experimental da ESALQ/USP localizada na Fazenda Areão, Piracicaba, SP (22 42’30’’ S; 47 38’30’’ W; 576 m). O clima é caracterizado como Cwa (mesotérmico úmido subtropical de inverno seco) de acordo com a classificação de Köppen. A precipitação pluvial média anual é de 1253 mm, com as chuvas concentradas no período de novembro a fevereiro. A temperatura média anual é de $21^{\circ} \mathrm{C}$, com a média do mês mais frio inferior a $18^{\circ} \mathrm{C}$ e a média do mês mais quente superior a $22^{\circ} \mathrm{C}$. A área se encontra cultivada com capim Tanzânia (Panicum maximum Jacq.), estabelecido em um Argissolo Vermelho eutrófico típico (Embrapa, 1999).

A área total do experimento é de 3,2 hectares, dividida em 8 blocos de 0,4 ha. Cada bloco contém 3 piquetes de 0,133 hectares, totalizando 24 piquetes. As áreas analisadas foram: uma irrigada com pivô central, e outra não irrigada, cada uma composta por 4 blocos e 12 piquetes (Figura 1).

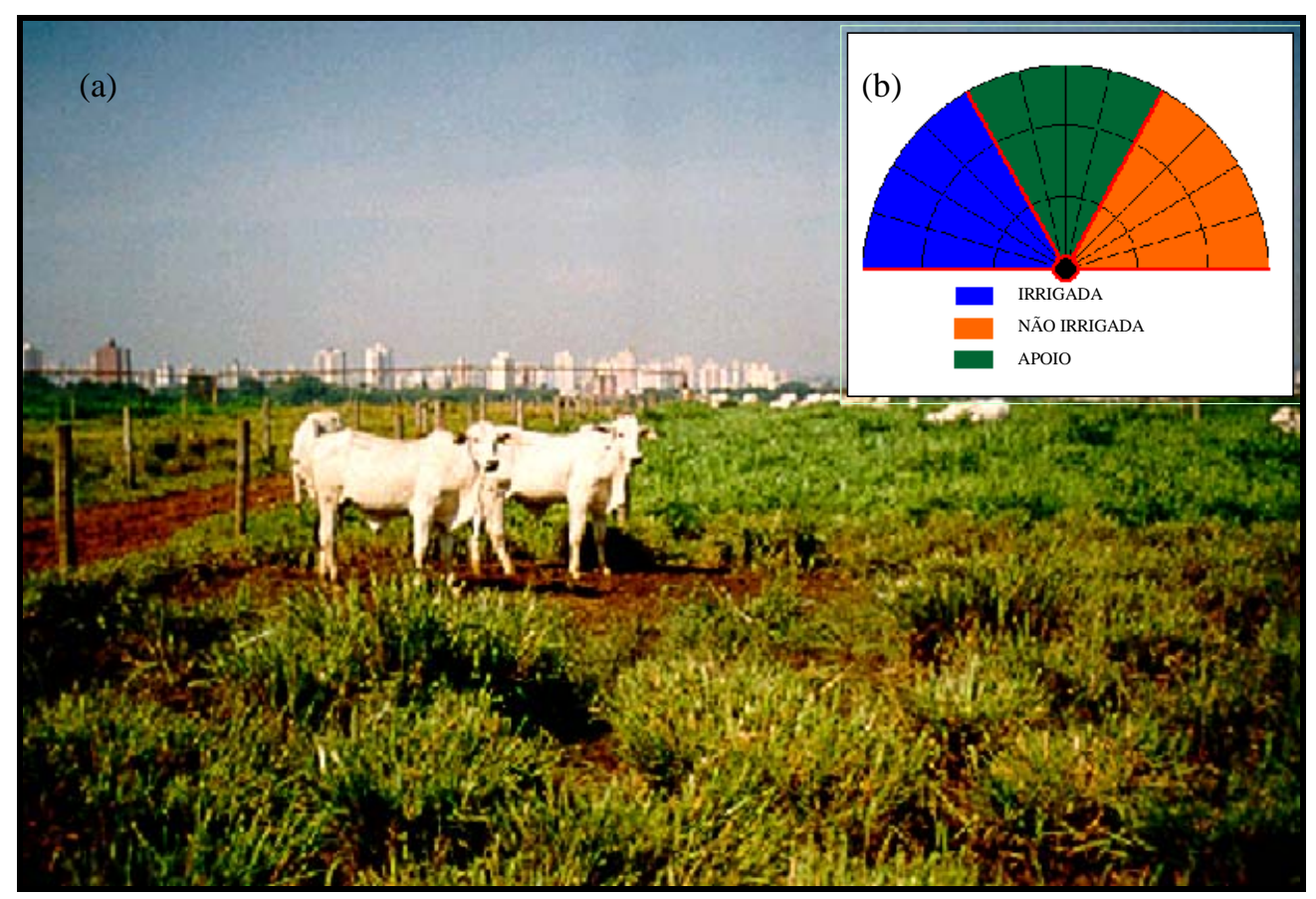

Figura 1 - (a) Fazenda Areão pertencente a ESALQ/USP e (b) Distribuição dos piquetes em cada área no experimento (irrigada, não irrigada e apoio) 
O ciclo do pastejo, os períodos de descanso e de ocupação foram de 36, 33 e 3 dias, respectivamente. A lotação animal foi ajustada em todos os piquetes para deixar uma quantidade de resíduo remanescente de $2500 \mathrm{~kg} \mathrm{MS} \mathrm{ha-1.}$

O preparo do solo consistiu de uma gradagem aradora e duas gradagens niveladoras. A semeadura foi realizada em linhas no mês de março de 1999. Para o estabelecimento das pastagens foi efetuada a correção do solo por meio de calagem e adubação. O primeiro pastejo foi realizado no mês de agosto do ano de 2001.

Para o manejo da irrigação foram instalados 5 baterias de tensiômetros nas profundidades de 10, 30, 50, 70 e 90 cm. A irrigação foi iniciada quando os tensiômetros registravam um potencial médio ( $\Psi$ ): - 30 a - $40 \mathrm{kPa}$ e finalizada em um potencial de

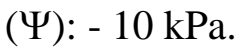

As amostragens foram feitas em 4 ciclos sucessivos correspondentes aos meses de novembro, dezembro, janeiro e fevereiro do ano de 2001 e 2002, respectivamente. Em cada piquete foi retirada, por ciclo, na camada superficial, uma amostra com estrutura indeformada, com anel volumétrico de $7 \mathrm{~cm}$ de diâmetro interno e 2,5 cm de altura, totalizando 96 amostras.

As amostras foram saturadas com água por 48 horas e equilibradas no potencial $(\psi)$ : - $10 \mathrm{kPa}$. Após o equilíbrio, cada amostra foi pesada e submetida ao ensaio de compressão uniaxial, que consistiu na aplicação sucessiva e contínua de pressões crescentes e preestabelecidas de 25, 50, 100, 200, 400, 600, 800, 1000, 1300 e 1600 kPa, por cinco minutos, período suficiente para atingir 99\% da deformação máxima (Silva et al., 2002). O deslocamento vertical a cada pressão aplicada foi registrado por meio de um sensor ligado a um sistema de aquisição de dados automatizado - MCT 2000 (MIRAE Engineering, Inc., Buscan, Korea). O ensaio permitiu a obtenção da curva de compressão do solo.

As amostras foram secadas em estufa a $105^{\circ} \mathrm{C}$, por 24 horas e a partir destes valores foi determinada para cada amostra a densidade do solo $\left(\mathrm{D}_{\mathrm{s}}\right)$ (Blake \& Hartge, 1986a). A partir das curvas de compressão uniaxial, foram obtidos os valores de pressão de preconsolidação (Casagrande, 1936) utilizando o software Mathcad (Mathsoft Inc., 2000) conforme Imhoff et al. (2004). 
Os valores médios da pressão de preconsolidação, para os sistemas de pastejo rotacionado intensivo irrigado $\left(\mathrm{PRI}_{\mathrm{I}}\right)$ e não irrigado $\left(\mathrm{PRI}_{\mathrm{NI}}\right)$, foram comparados pelo teste que considera a diferença mínima significativa a 5\%, utilizando o software SAS (Statistical Analysis System Institute, 1991).

\subsection{Resultados e Discussão}

Os momentos estatísticos das variáveis analisadas sob os sistemas de pastejo rotacionado intensivo irrigado $\left(\mathrm{PRI}_{\mathrm{I}}\right)$ e não irrigado $\left(\mathrm{PRI}_{\mathrm{NI}}\right)$ são apresentados na Tabela 1.

A amplitude de variação da umidade com base gravimétrica esteve possivelmente relacionada com o intervalo de variação do teor argila em cada área de pastejo.

A pressão de preconsolidação, considerada um indicador da pressão máxima a que o solo foi submetido anteriormente apresentou, nos dois sistemas, valores médios semelhantes aos obtidos por Kondo \& Dias Júnior (1999) em solos sob pastagens. Por outro lado, foram superiores aos valores encontrados por Silva et al. (2002) em um solo sob as culturas de soja e de milho. Estas diferenças podem ser atribuídas ao fato do gado aplicar elevadas pressões ao solo, podendo superar os valores de pressões aplicadas por máquinas agrícolas convencionais (Mapfumo et al., 1999), e à ausência de revolvimento na camada superficial do solo cultivado com pastagens, operação que normalmente ocorre no cultivo de culturas anuais.

O intervalo de variação da pressão de preconsolidação nas duas áreas analisadas foi semelhante aos valores da pressão exercida por animais da raça bovina, indicados na literatura científica (Willatt \& Pullar, 1983; Betterige et al., 1999).

O intervalo de variação da densidade do solo indicou ampla variabilidade espacial do estado de compactação, provavelmente ocasionada pelas condições diferenciadas de umidade nos dois sistemas $\left(\mathrm{PRI}_{\mathrm{I}}\right.$ e $\left.\mathrm{PRI}_{\mathrm{NI}}\right)$. O maior valor médio de densidade observado no sistema de $\mathrm{PRI}_{\mathrm{I}}$ reflete o efeito do teor de umidade sobre a compactação do solo. Um aumento de densidade, ocasionado pelo maior teor de 
umidade do solo no momento do pisoteio do gado também foi observado por Ferrero (1991), Proffitt et al. (1993) e Jorajúria et al. (1997).

Tabela 1. Momentos estatísticos das variáveis analisadas sob os sistemas de pastejo rotacionado intensivo irrigado $\left(\mathrm{PRI}_{\mathrm{I}}\right)$ e não irrigado $\left(\mathrm{PRI}_{\mathrm{NI}}\right)$

\begin{tabular}{|c|c|c|c|c|c|c|}
\hline Variáveis ${ }^{(1)}$ & $\mathrm{N}$ & Média & $\begin{array}{l}\text { Desvio } \\
\text { padrão }\end{array}$ & Mínimo & Máximo & $\mathrm{CV}, \%$ \\
\hline & \multicolumn{6}{|c|}{$\mathrm{PRI}_{\mathrm{I}}$} \\
\hline Argila, g kg-1 & 12 & 425,83 & 32,87 & 370,00 & 480,00 & 7,72 \\
\hline Areia, $\mathrm{g} \mathrm{kg}^{-1}$ & 12 & 329,17 & 47,12 & 250,00 & 410,00 & 14,32 \\
\hline Silte, $\mathrm{g} \mathrm{kg}^{-1}$ & 12 & 235,00 & 34,39 & 190,00 & 290,00 & 14,64 \\
\hline $\mathrm{Ug}, \mathrm{kg} \mathrm{kg}^{-1}$ & 48 & 0,18 & 0,01 & 0,15 & 0,20 & 6,77 \\
\hline$\sigma_{\mathrm{p}}, \mathrm{kPa}$ & 48 & 268,00 & 86,49 & 107,00 & 466,00 & 32,25 \\
\hline \multirow[t]{2}{*}{$\mathrm{D}_{\mathrm{s}}, \mathrm{Mg} \mathrm{m}^{-3}$} & 48 & 1,68 & 0,08 & 1,53 & 1,83 & 4,62 \\
\hline & \multicolumn{6}{|c|}{$\mathrm{PRI}_{\mathrm{NI}}$} \\
\hline Argila, g kg-1 & 12 & 517,50 & 42,50 & 440,00 & 580,00 & 8,21 \\
\hline Areia, g kg-1 & 12 & 267,50 & 32,26 & 220,00 & 330,00 & 12,06 \\
\hline Silte, $\mathrm{g} \mathrm{kg}^{-1}$ & 12 & 216,67 & 20,97 & 180,00 & 250,00 & 9,91 \\
\hline Ug, kg kg ${ }^{-1}$ & 48 & 0,22 & 0,02 & 0,15 & 0,25 & 11,20 \\
\hline$\sigma_{\mathrm{p}}, \mathrm{kPa}$ & 48 & 246,00 & 82,12 & 104,00 & 438,00 & 33,44 \\
\hline $\mathrm{D}_{\mathrm{s}}, \mathrm{Mg} \mathrm{m}^{-3}$ & 48 & 1,61 & 0,08 & 1,45 & 1,75 & 4,83 \\
\hline
\end{tabular}

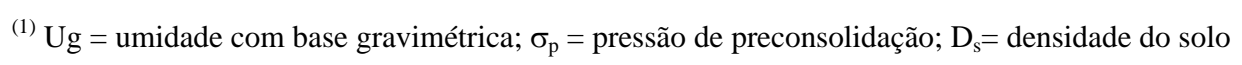

Os valores médios da $\sigma_{\mathrm{p}}$ em cada ciclo, nos sistemas de $\mathrm{PRI}_{\mathrm{I}}$ e $\mathrm{PRI}_{\mathrm{NI}}$ estão apresentados na Figura 2. A sobreposição das barras verticais indica a ausência de diferença significativa ao nível de 5\%. Diferenças são consideradas significativas na ausência de sobreposição das barras verticais (Andrews et al., 1980). 


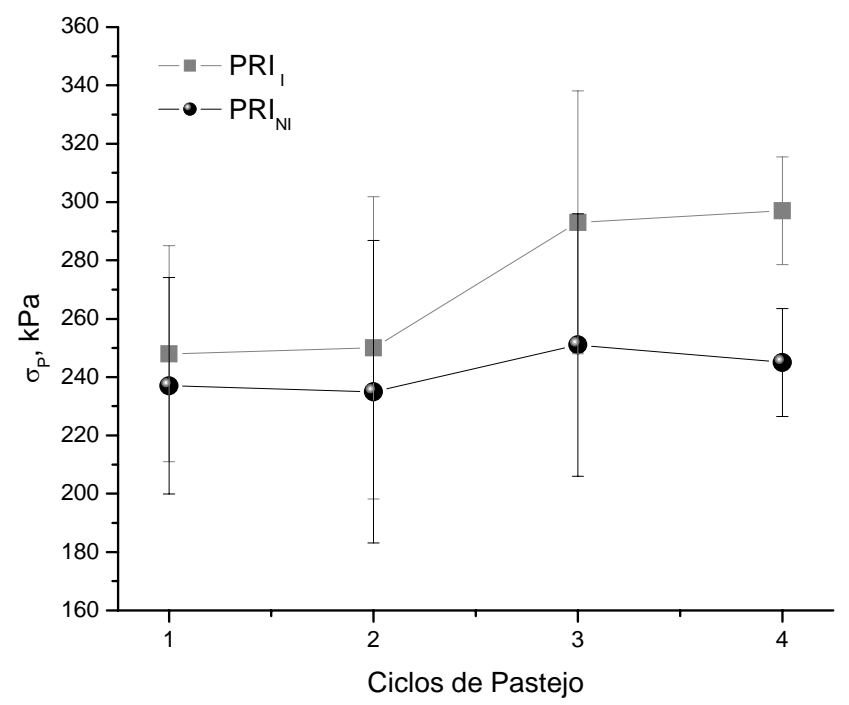

Figura 2 - Valores médios da pressão de preconsolidação do solo $\left(\sigma_{\mathrm{p}}\right)$ em cada ciclo sob os sistemas de pastejo rotacionado intensivo irrigado $\left(\mathrm{PRI}_{\mathrm{I}}\right)$ e não irrigado $\left(\mathrm{PRI}_{\mathrm{NI}}\right)$. Barras verticais indicam a diferença mínima significativa a $5 \%$ entre os sistemas de $P R I_{I}$ e $P R I_{N I}$

Os valores da $\sigma_{\mathrm{p}}$ foram maiores no sistema de $\mathrm{PRI}_{\mathrm{I}}$, indicando que o solo foi submetido a um maior estresse nesse sistema. A umidade do solo no momento do pisoteio, mais elevada nesse sistema, pode ter contribuído para o aumento da compactação no sistema de $\mathrm{PRI}_{\mathrm{I}}$ quando comparado ao sistema de $\mathrm{PRI}_{\mathrm{NI}}$. O conteúdo de água do solo determina a magnitude de deformação, para um idêntico valor de pressão aplicada, como assinalado por Larson \& Gupta (1980).

Os animais aplicam elevadas pressões no solo porque o seu peso é distribuído numa área (o casco) muito pequena. Além disso, os animais não permanecem estáticos na área de pastejo. A pressão que os animais exercem sobre o solo, também pode estar relacionada à energia cinética que é transmitida ao solo, quando estes estão em movimento (Willatt \& Pullar, 1983). Um animal em movimento é capaz de realizar um deslocamento ou uma deformação em um outro corpo (solo). A força devido a este movimento é chamada de energia cinética, a qual está diretamente relacionada a massa (peso do animal) e a velocidade de movimentação. Abaye et al. (1997) indicaram que a 
força dinâmica exercida por um animal sobre o solo é aproximadamente o dobro da força estática, o que corrobora as observações de Nie et al. (2001).

A ausência de diferença significativa da $\sigma_{\mathrm{p}}$ até o terceiro ciclo de pastejo pode estar relacionada à variabilidade dos dados. A diminuição da variabilidade da $\sigma_{\mathrm{p}}$ no decorrer do tempo, avaliada por meio do coeficiente de variação, justifica a obtenção de diferenças significativas deste parâmetro entre os dois sistemas analisados no último ciclo de pastejo (Figura 3).

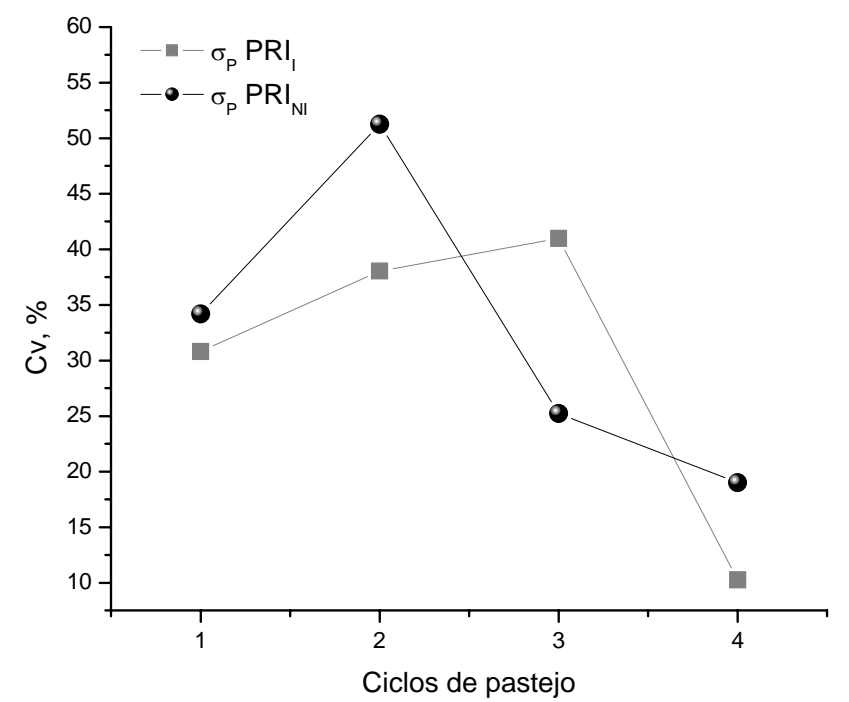

Figura 3 - Coeficiente de variação da $\sigma_{\mathrm{p}}$ em cada ciclo sob os sistemas de pastejo rotacionado intensivo irrigado $\left(\mathrm{PRI}_{\mathrm{I}}\right)$ e não irrigado $\left(\mathrm{PRI}_{\mathrm{NI}}\right)$

A variabilidade da $\sigma_{\mathrm{p}}$ pode estar associada ao efeito do pisoteio dos animais e ao tempo de avaliação do experimento. Em função dos animais não permanecerem estáticos na área, é comum observar em sistemas de pastejo, pontos mais compactados (correspondentes às marcas dos cascos) distribuídos desuniformemente no solo. Essa desuniformidade resulta incrementada quando o solo está úmido, o que corrobora os valores observados no sistema irrigado.

Por outro lado, é de se esperar que com os sucessivos ciclos de pastejo, os animais pisoteiem toda a área pastejada, induzindo à maior compactação e uniformidade 
do estado físico do solo, contribuindo assim para a redução da variabilidade e obtenção de diferenças significativas dos parâmetros analisados. Os resultados indicam que, em seis meses de implantação do sistema de pastejo, ocorreu uma diminuição da variabilidade e uma tendência de homogeneização do estado de compactação da área. Similar tendência dos valores de capacidade de suporte de carga avaliada no decorrer de um experimento foi apresentada por Horn, (2003).

Uma grande variabilidade também foi verificada nos valores de densidade do solo em cada ciclo de pastejo (Figura 4).

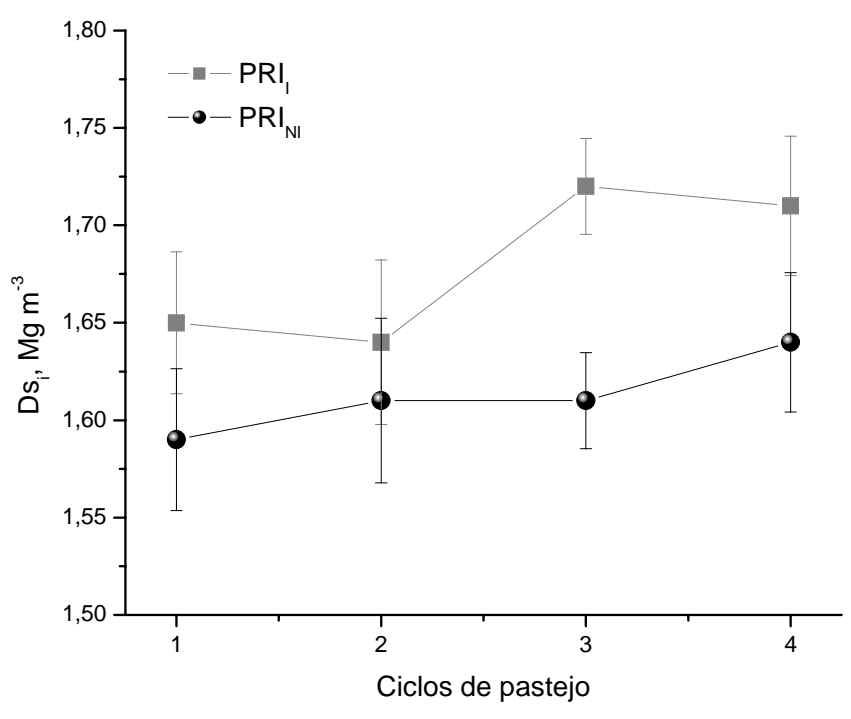

Figura 4 - Valores médios de densidade do solo $\left(\mathrm{Ds}_{\mathrm{i}}\right)$ em cada ciclo sob os sistemas de pastejo rotacionado intensivo irrigado $\left(\mathrm{PRI}_{\mathrm{I}}\right)$ e não irrigado $\left(\mathrm{PRI}_{\mathrm{NI}}\right)$. Barras verticais indicam a diferença mínima significativa a 5\% entre os sistemas de $\mathrm{PRI}_{\mathrm{I}}$ e $\mathrm{PRI}_{\mathrm{NI}}$

Vários estudos indicam que a $\sigma_{\mathrm{p}}$ é influenciada pela densidade do solo (Silva et al., 2000; Canarache et al., 2000; Silva et al., 2002; Imhoff et al., 2004). A redução da variabilidade dos dados de densidade do solo condicionou a obtenção de diferenças significativas no terceiro ciclo de pastejo. Foi possível perceber uma homogeneização nos valores deste indicador com o decorrer do tempo do experimento, corroborando os dados de Bell et al. (1997) e de Twerdoff et al. (1999) em sistemas pastoris. 
O modelo não linear proposto por McNabb \& Boersma (1993) foi o que melhor ajustou os dados das curvas de compressão nos sistemas avaliados. Os resultados são apresentados na Tabela 2.

Tabela 2. Resultados da regressão não linear para o modelo: $\ln \rho=\ln \left(\rho_{0} * \delta_{\mathrm{i}}\right)-(\mathrm{a}+\mathrm{b} * \sigma$ $\left.+\mathrm{c} * \delta_{\mathrm{c}}\right) *\left(1-\mathrm{e}^{-\mathrm{d}^{*} \sigma}\right)$ sob os sistemas de pastejo rotacionado intensivo irrigado $\left(\mathrm{PRI}_{\mathrm{I}}\right)$ e não irrigado $\left(\mathrm{PRI}_{\mathrm{NI}}\right)$

\begin{tabular}{|c|c|c|c|c|}
\hline \multirow[b]{2}{*}{ Parâmetros } & \multirow[b]{2}{*}{ Valor estimado } & \multirow[b]{2}{*}{ Erro Padrão } & \multicolumn{2}{|c|}{ Intervalo de confiança } \\
\hline & & & Limite inferior & Limite superior \\
\hline & \multicolumn{4}{|c|}{$\mathrm{PRI}_{\mathrm{I}}{ }^{(1)}$} \\
\hline$\rho_{0}$ & 1,683 & 0,004 & 1,675 & 1,692 \\
\hline $\mathrm{a}$ & $-0,072$ & 0,006 & $-0,0843$ & $-0,059$ \\
\hline $\mathrm{b}$ & $-0,045$ & 0,005 & $-0,056$ & $-0,034$ \\
\hline c & 5,547 & 0,961 & 3,658 & 7,436 \\
\hline \multirow[t]{2}{*}{$\mathrm{d}$} & 0,229 & 0,021 & 0,189 & 0,271 \\
\hline & \multicolumn{4}{|c|}{$\mathrm{PRI}_{\mathrm{NI}}{ }^{(2)}$} \\
\hline$\rho_{o}$ & 1,618 & 0,003 & 1,612 & 1,624 \\
\hline $\mathrm{a}$ & $-0,076$ & 0,005 & $-0,086$ & $-0,066$ \\
\hline $\mathrm{b}$ & $-0,049$ & 0,004 & $-0,058$ & $-0,041$ \\
\hline c & 5,134 & 0,659 & 3,838 & 6,430 \\
\hline $\mathrm{d}$ & 0,204 & 0,014 & 0,176 & 0,232 \\
\hline $\begin{array}{l}\rho=\text { densidade do so } \\
\sigma=\text { pressão aplicado } \\
{ }^{(1)} \mathrm{F}=62581,1 ; \mathrm{P}< \\
{ }^{(2)} \mathrm{F}=103147 ; \mathrm{P}<\mathrm{C}\end{array}$ & $\begin{array}{l}\left.\text { Ig m }{ }^{-3}\right) ; \delta_{\mathrm{i}}=\text { densidade } \\
1 ; \mathrm{R}^{2}=0,88(\mathrm{~N}=440 \\
1 ; \mathrm{R}^{2}=0,94(\mathrm{~N}=440\end{array}$ & al/densidade méc & $=\left(\delta_{\mathrm{i}}-1\right) \times($ densid & $\left.0 \mathrm{MPa}, \mathrm{Mg} \mathrm{m}^{-3}\right)$ \\
\hline
\end{tabular}

O modelo explicou 88 e 94\% da variabilidade dos dados de densidade do solo nos sistemas de $\mathrm{PRI}_{\mathrm{I}}$ e $\mathrm{PRI}_{\mathrm{NI}}$, respectivamente. Todos os parâmetros foram significativos $(\mathrm{P}<0,0001)$, pois o intervalo de confiança do modelo não incluiu o valor zero (Tabela 2).

A partir do modelo, foram construídas as curvas de compressão estimadas para os sistemas de $\mathrm{PRI}_{\mathrm{I}}$ e $\mathrm{PRI}_{\mathrm{NI}}$ (Figura 5). 
As curvas de compressão uniaxial do solo refletem as diferenças no processo de compactação dos solos em cada área de pastejo. Esta diferença é observada no deslocamento da curva de compressão uniaxial para valores superiores de densidade do solo no sistema de $\mathrm{PRI}_{\mathrm{I}}$. O deslocamento no sistema de $\mathrm{PRI}_{\mathrm{I}}$ pode ter sido condicionado ao maior teor de umidade do solo no momento do pisoteio do gado.

A umidade do solo é o fator mais importante que determina a magnitude de deformação que pode ocorrer no solo quando determinado estresse é aplicado (McNabb \& Boersma, 1996; Sanches-Girón et al., 1998; Mosaddeghi et al., 2000), já que a água atua como lubrificante entre as partículas do solo favorecendo o deslocamento e rearranjamento das mesmas para posições de maior proximidade (maior densidade do solo).

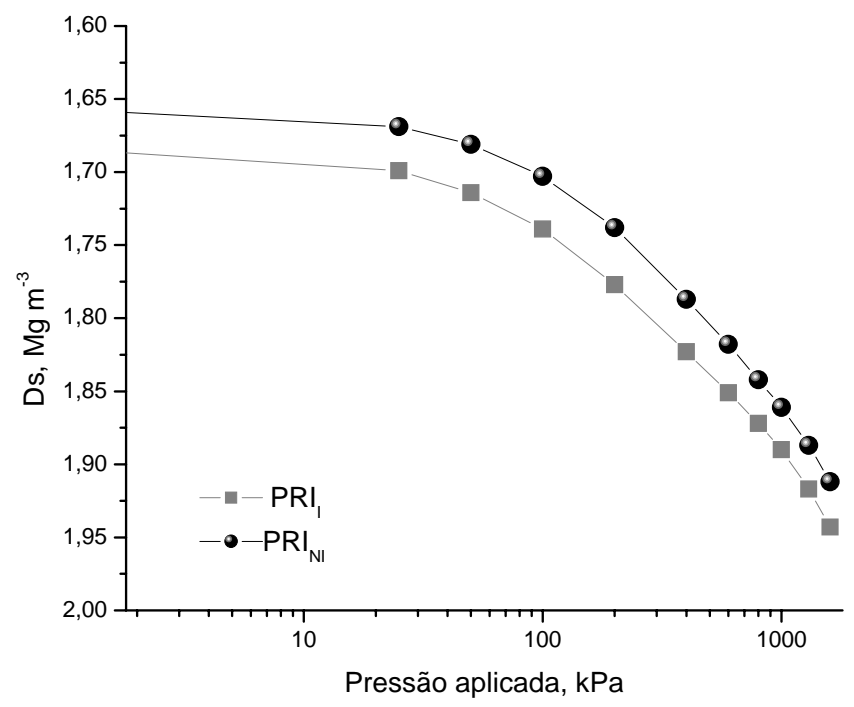

Figura 5 - Curvas médias de compressão uniaxial estimadas sob os sistemas de pastejo rotacionado intensivo irrigado ( $\left(\mathrm{PRI}_{\mathrm{I}}\right)$ e não irrigado $\left(\mathrm{PRI}_{\mathrm{NI}}\right)$

\subsection{Conclusões}

Os resultados comprovam a hipótese de que há diferença no processo de compactação do solo sob os sistemas de pastejo rotacionado intensivo irrigado e não irrigado. 
As curvas de compressão foram deslocadas para valores superiores de densidade do solo no sistema de pastejo rotacionado intensivo irrigado. O aumento da compactação do solo com os sucessivos ciclos de pastejo é corroborado pelos valores de pressão de preconsolidação que foram significativamente maiores no quarto ciclo de pastejo no sistema de pastejo rotacionado intensivo irrigado em relação ao sistema não irrigado.

O sistema de pastejo rotacionado intensivo irrigado pode induzir a degradação física do solo se a técnica de irrigação não for adequadamente conduzida. 


\section{ESTIMATIVA DA CAPACIDADE DE SUPORTE DE CARGA DO SOLO A PARTIR DA AVALIAÇÃO DA RESISTÊNCIA À PENETRAÇÃO}

\section{Resumo}

A pressão de preconsolidação, apesar das dificuldades inerentes à sua obtenção, provê de uma medida útil do estado mecânico do solo para o seu uso, manejo e planejamento de sistemas de mecanização em áreas citrícolas. A estimativa da pressão de preconsolidação a partir de propriedades do solo facilmente mensuráveis é, portanto, de relevância para solos tropicais. Os objetivos desse trabalho foram: (i) quantificar a pressão de preconsolidação, (ii) quantificar a resistência do solo à penetração em laboratório e à campo e (iii) estabelecer a relação entre ambos os indicadores físicos em um Latossolo Vermelho Amarelo sob pomar de laranja. A amostragem e as avaliações foram realizadas em 8 dias consecutivos acompanhando o secamento natural do solo. Foi avaliada a resistência do solo à penetração em campo e em laboratório e a pressão de preconsolidação a partir da curva de compressão uniaxial. Houve uma relação significativa, positiva e linear entre a pressão de preconsolidação e a resistência do solo à penetração medida no laboratório e no campo. Os resultados indicaram que a capacidade de suporte de carga pode ser estimada a partir de uma propriedade física facilmente mensurável como a resistência do solo à penetração em solos sob clima tropical.

Palavras-chave: pomar de laranja, resistência do solo à penetração, pressão de preconsolidação, Latossolo. 


\section{ESTIMATE OF LOAD SUPPORT CAPACITY FROM THE AVALIATION OF SOIL RESISTANCE TO PENETRATION}

\section{Summary}

The preconsolidation pressure, despite the difficulties inherent to its measurement, provides an useful measure of the soil mechanical state for the soil management, and the design of mechanization systems in orchards. The estimation of the preconsolidation pressure using easily measurable soil physical properties is relevant for tropical soils. The objectives of this study were: (i) to quantify the preconsolidation pressure (ii) to quantify the soil resistance to penetration measured in the laboratory and the field, and (iii) to establish the relationships between these physical indicators in an Haplustox under orange orchard. The sampling and the evaluations were accomplished in 8 consecutive days according the soil natural drying. The soil resistance to penetration was evaluated in field and in laboratory, and the preconsolidation pressure was obtained from the uniaxial compression curve. There was a positive, linear, and significant relationship between the preconsolidation pressure and the soil resistance to penetration measured in the laboratory as well as in the field. The results indicated that the load support capacity can be estimated from an easily measurable soil physical property; i.e. the soil resistance to penetration, in soils under tropical climate.

Key words: orange orchard, soil resistance to penetration, preconsolidation pressure, Haplustox.

\subsection{Introdução}

Nas últimas três décadas uma considerável quantidade de pesquisas têm sido desenvolvidas com relação ao processo de compressão do solo (Earl, 1997). O processo de compressão descreve a compactação (expulsão do ar) e a consolidação (expulsão da água) do solo (Gupta \& Allmaras, 1987). O entendimento do processo de compressão é essencial para estimar as alterações que podem ocorrer na estrutura quando o solo é submetido à determinada pressão externa. 
A curva de compressão tem sido amplamente utilizada para compreender o processo de compressão do solo. A partir desta curva é obtido um indicador que expressa a máxima pressão que pode ser aplicada ao solo antes da ocorrência de um incremento substancial na compactação. Na literatura, este indicador é chamado de pressão de preconsolidação, sendo considerado um valor crítico, já que pressões inferiores a este valor causam pouca compactação adicional, enquanto que estresses maiores induzem considerável compactação adicional ao solo originando a degradação irreversível da estrutura (Dawidowski et al., 2001). Por este motivo a pressão de preconsolidação é considerada uma estimativa da história do estresse à qual o solo foi submetido no passado e de sua capacidade de suporte de carga (Veenhof \& McBride, 1996).

A determinação da pressão de preconsolidação é um processo demorado e está associada à aquisição de equipamentos de custo elevado. Exige a construção da curva de compressão que baseia-se na aplicação de pressões sucessivas a intervalos regulares de tempo. Posteriormente, a obtenção da pressão de preconsolidação pode ser realizada utilizando diferentes metodologias (Dias Júnior \& Pierce, 1995). Estas metodologias incluem procedimentos gráficos (Casagrande, 1936), o emprego de técnicas de regressão (Lebert \& Horn, 1991; Assouline et al., 1997; Fritton, 2001) e, mais recentemente, a determinação tem sido feita com auxílio de softwares com programas específicos (Veenhof \& McBride, 1996, Fritton, 2001, Imhoff et al., 2004).

A importância do conhecimento da pressão de preconsolidação é amplamente reconhecida por auxiliar na implementação de sistemas de manejo conservacionistas e estar relacionada a resistência do solo à penetração das raízes (Römkens \& Miller, 1971). Estes autores indicaram que a pressão de preconsolidação é uma estimativa da resistência do solo na qual a elongação das raízes cessa, sugerindo que solos com valores elevados de pressão de preconsolidação apresentam maior probabilidade de reduzir o crescimento das raízes.

Uma estimativa da pressão de preconsolidação obtida a partir de propriedades físicas facilmente mensuráveis, como a resistência do solo à penetração, provê de uma 
medida útil do estado mecânico do solo para o seu uso, manejo e planejamento de sistemas de mecanização agrícola.

Culley \& Larson (1987) encontraram uma relação linear entre a resistência do solo à penetração e a pressão de preconsolidação. Burguer et al. (1988) mostraram que a variação na pressão de preconsolidação com a profundidade é comparável com a variação da resistência do solo à penetração. Alguns autores têm mencionado que existe uma relação entre a RP e a $\sigma_{P}$ de 10:1 (Canarache et al., 2000). Recentemente esta relação foi confirmada por Mosaddeghi et al. (2003) para solos de clima temperado.

A hipótese testada no presente trabalho foi de que a capacidade de suporte de carga pode ser estimada a partir de avaliações da resistência do solo à penetração em solos de clima tropical. Os objetivos foram: (i) quantificar a pressão de preconsolidação $\left(\sigma_{\mathrm{P}}\right)$, (ii) quantificar a resistência do solo à penetração em laboratório $\left(\mathrm{RP}_{\mathrm{l}}\right)$ e à campo $\left(\mathrm{RP}_{\mathrm{c}}\right)$ e (iii) estabelecer a relação entre ambos os indicadores físicos em um Latossolo Vermelho Amarelo sob pomar de laranja.

\subsection{Material e Métodos}

O estudo foi desenvolvido em um pomar de laranja pêra (Citrus sinensis (L.) Osbeck) sobre o porta-enxerto limão “cravo” (Citrus limonia (L.) Osbeck), com uma área total de $24.128 \mathrm{~m}^{2}$ implantado em 1991 e pertencente ao Departamento de Produção Vegetal (Setor de Horticultura) da ESALQ/USP, localizado na cidade de Piracicaba, SP. O solo é classificado como Latossolo Vermelho Amarelo distrófico (Embrapa, 1999) de textura franco arenosa ( $80 \mathrm{~g} \mathrm{~kg}^{-1}$ de silte, $750 \mathrm{~g} \mathrm{~kg}^{-1}$ de areia e $170 \mathrm{~g} \mathrm{~kg}^{-1}$ de argila).

A amostragem foi realizada no mês de junho do ano de 2003. Na área de amostragem foram estabelecidas cinco parcelas, as quais foram irrigadas até atingir a umidade de saturação na camada superficial $(0$ a $10 \mathrm{~cm})$. A partir do segundo dia foram coletadas 5 amostras com estrutura indeformada (uma por parcela) por 8 dias consecutivos, acompanhando o secamento natural do solo, totalizando 40 amostras. As amostras, coletadas utilizando anéis volumétricos com altura de 2,5 cm e diâmetro 
interno de 7,0 cm, corresponderam a uma profundidade média de amostragem de 5 a 7,5 $\mathrm{cm}$.

Imediatamente antes da coleta das amostras com estrutura indeformada e durante os oito dias foi avaliada a resistência do solo à penetração em campo realizando cinco medidas ao redor do local em que seria coletada cada amostra indeformada na profundidade de 0 a $8 \mathrm{~cm}$, utilizando-se um penetrômetro estático convencional cônico, modelo Solotest S-310, com ângulo de penetração de $30^{\circ}$ e área basal de $1 \mathrm{~cm}^{2}$, totalizando 200 leituras.

As amostras foram levadas ao laboratório para a determinação da resistência do solo à penetração e da curva de compressão uniaxial. Foram pesadas e imediatamente obtidas três repetições de resistência do solo à penetração em cada amostra com estrutura indeformada empregando-se um equipamento que utiliza um atuador linear e célula de carga de $20 \mathrm{~kg}$ acoplado a um microcomputador para a aquisição e armazenamento das informações, perfazendo um total de 120 avaliações.

Após a avaliação da resistência do solo à penetração as amostras foram recobertas para manter a umidade até o momento da realização do ensaio de compressão uniaxial. Este ensaio consistiu na aplicação sucessiva e contínua de pressões crescentes e preestabelecidas de 25, 50, 100, 200, 400, 600, 800, 1000, 1300 e $1600 \mathrm{kPa}$, por cinco minutos, período suficiente para atingir 99\% da deformação máxima (Silva et al., 2002). O deslocamento vertical a cada pressão aplicada foi registrado por meio de um sensor ligado a um sistema de consolidação e aquisição de dados automatizado Satron MCT 2000 (MIRAE Engineering Inc., Buscan Korea).

As amostras foram secadas em estufa a $105^{\circ} \mathrm{C}$, por 24 horas e a massa de cada amostra foi determinada. A partir dos valores das massas e do volume calculado para cada pressão aplicada foram determinados os valores de densidade do solo para cada amostra (Blake \& Hartge, 1986a).

A densidade de partículas foi determinada pelo método do picnômetro (Blake \& Hartge, 1986b). A relação de vazios (e) foi determinada para cada amostra baseando-se na densidade do solo $\left(D_{s}\right)$ e na densidade de partículas $\left(D_{p}\right)\left(e=\left(D_{p} / D_{s}\right)-1\right)($ McBride \& Joosse, 1996). 
O ensaio permitiu a obtenção da curva de compressão uniaxial do solo, que relaciona o logaritmo da pressão aplicada (eixo x) versus a relação de vazios (eixo y). A partir destas curvas, foi obtido o valor da pressão de preconsolidação $\left(\sigma_{p}\right)$ (Imhoff et al., 2004).

Os resultados foram submetidos à analises de regressão linear e não linear utilizando o software SAS (Statistical Analysis System Institute, 1991).

\subsection{Resultados e Discussão}

Os momentos estatísticos das variáveis analisadas são apresentados na Tabela 1. O secamento natural do solo nos oito dias consecutivos de amostragem condicionou uma amplitude de variação de umidade. O intervalo de variação da densidade do solo $\left(\mathrm{D}_{\mathrm{s}}\right)$ pode estar associado ao tráfego de máquinas para executar as operações agrícolas de adubação, pulverização, controle de plantas daninhas e colheita no pomar.

Tabela 1. Momentos estatísticos das variáveis analisadas

\begin{tabular}{ccccccc}
\hline Variáveis $^{(1)}$ & $\mathrm{N}$ & Média & $\begin{array}{c}\text { Desvio } \\
\text { padrão }\end{array}$ & Mínimo & Máximo & $\mathrm{CV}(\%)$ \\
\hline $\mathrm{Ug}$ & 40 & 0,11 & 0,02 & 0,08 & 0,17 & 21,44 \\
$\mathrm{D}_{\mathrm{s}}$ & 40 & 1,52 & 0,08 & 1,40 & 1,69 & 5,45 \\
$\mathrm{RP}_{\mathrm{l}}$ & 40 & 2099 & 1045,21 & 700 & 4763 & 49,79 \\
$\mathrm{RP}_{\mathrm{c}}$ & 40 & 2056 & 1175,69 & 568 & 4743 & 57,17 \\
$\sigma_{\mathrm{p}}$ & 40 & 121 & 67 & 38 & 251 & 55,16 \\
\hline
\end{tabular}

${ }^{(1)} \mathrm{Ug}=$ umidade gravimétrica $\left(\mathrm{kg} \mathrm{kg}^{-1}\right) ; \mathrm{D}_{\mathrm{s}}=$ densidade inicial $\left(\mathrm{Mg} \mathrm{m}^{-3}\right) ; \mathrm{RP}_{\mathrm{c}}$ e $\mathrm{RP}_{\mathrm{l}}(\mathrm{kPa})$ = resistência do solo à penetração avaliada em laboratório e à campo, respectivamente; $\sigma_{\mathrm{p}}=$ pressão de preconsolidação $(\mathrm{kPa})$

A amplitude de variação da resistência do solo à penetração avaliada em laboratório $\left(\mathrm{RP}_{\mathrm{l}}\right)$, à campo $\left(\mathrm{RP}_{\mathrm{c}}\right)$ e da pressão de preconsolidação $\left(\sigma_{\mathrm{P}}\right)$ esteve associada provavelmente com a variação da umidade e da $\mathrm{D}_{\mathrm{s}}$. Estudos apresentam relações entre a 
resistência do solo à penetração (RP) e a $\mathrm{D}_{\text {s }}$ (Gerard et al., 1982; Prado et al., 2002; Lampurlanés \& Cantero-Martínez, 2003). Os autores evidenciam um aumento da RP com o incremento da densidade do solo. Da mesma forma, estudos mostram uma relação positiva entre a $\sigma_{\mathrm{P}}$ e a $\mathrm{D}_{\mathrm{s}}$ (Salire et al., 1994; Alexandrou \& Earl, 1998; Silva et al., 2002; Imhoff et al., 2004).

A variação dos indicadores físicos avaliados (RP e $\sigma_{\mathrm{P}}$ ) também está associada com o intervalo de variação da umidade com base gravimétrica imposta ao solo neste estudo. As Figuras 1 e 2 mostram os valores de $\mathrm{RP}_{\mathrm{l}}$, de $\mathrm{RP}_{\mathrm{c}}$ e da $\sigma_{\mathrm{P}}$ em função da variação da umidade com base gravimétrica. Todas as variáveis analisadas foram significativamente relacionadas com a umidade do solo $(\mathrm{P}<0,0001)$.

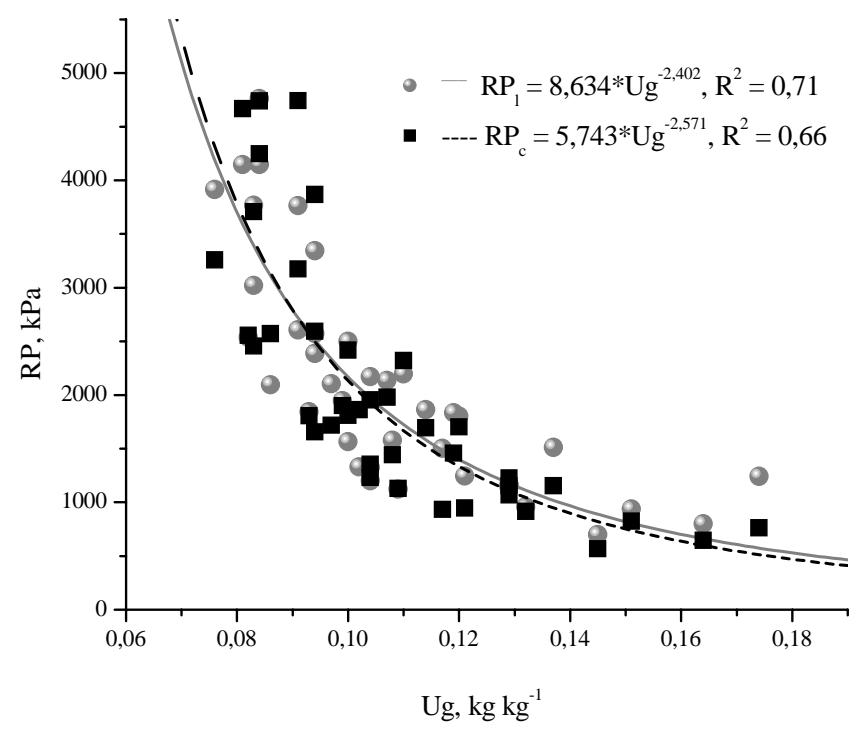

Figura 1 - Resistência do solo à penetração avaliada em laboratório $\left(\mathrm{RP}_{\mathrm{l}}\right)$ e à campo $\left(\mathrm{RP}_{\mathrm{c}}\right)$ versus umidade gravimétrica $(\mathrm{Ug})$ na área de citrus

Estudos têm indicado que a RP diminui com o incremento da umidade do solo (Taylor et al., 1966; Häkansson et al., 1988; Busscher et al., 1997; Vaz \& Hopmans, 2001). Da mesma forma, diversos trabalhos mostram que a $\sigma_{P}$ diminui com o aumento 
da umidade do solo (Horn, 1988; Alexandrou \& Earl, 1998; Kondo \& Dias Júnior, 1999). A umidade é um fator amplamente reconhecido como determinante do processo de compressão e que determina a magnitude da deformação de um determinado tipo de solo. Atua como lubrificante entre as partículas permitindo o deslocamento das mesmas. Este deslocamento é favorecido à medida que há incremento da umidade do solo (Larson et al., 1980; McNabb et al., 2001; Sánchez-Girón et al., 2001).

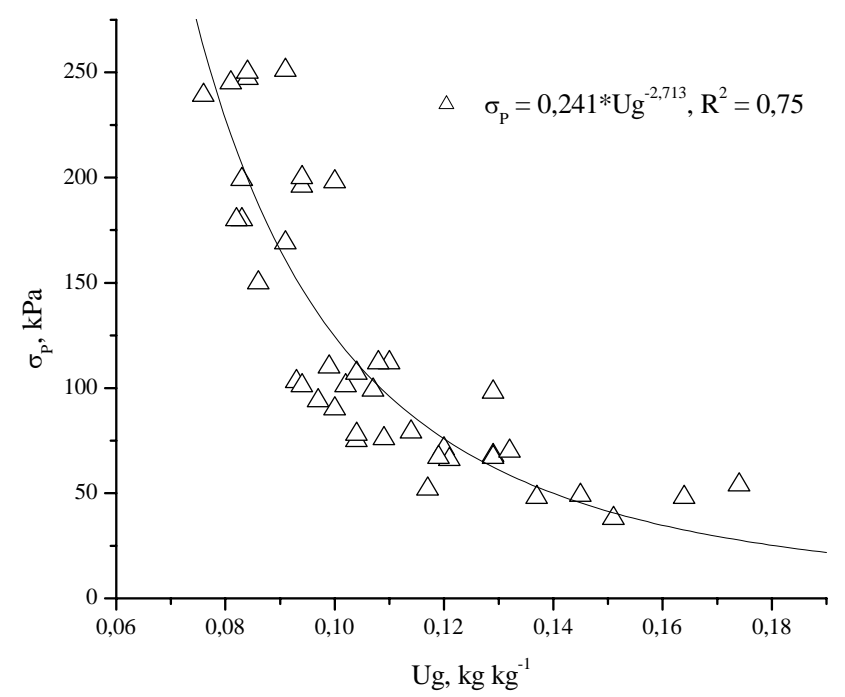

Figura 2 - Pressão de preconsolidação $\left(\sigma_{\mathrm{P}}\right)$ versus umidade gravimétrica (Ug) na área de citrus

O incremento da umidade origina uma redução do número de contatos entre as partículas diminuindo as forças de ligação entre elas e por sua vez reduzindo a capacidade de suporte de carga do solo (Horn \& Lebert, 1994; McNabb \& Boersma, 1996; Silva et al., 2002, Imhoff et al., 2004).

Devido ao fato de ambas as variáveis analisadas dependerem das mesmas propriedades, espera-se que exista uma relação entre elas. Estudos têm sido feitos no sentido de mostrar a relação existente entre a RP e a $\sigma_{\mathrm{P}}$. Os autores observaram uma 
relação de 10:1 entre a RP e a $\sigma_{\mathrm{P}}$ (Farrell \& Greacen, 1966; Canarache et al., 2000; Mosaddeghi et al., 2003).

Na Tabela 2 é mostrada a análise de regressão da $\sigma_{\mathrm{P}}$ em função da $\mathrm{RP}_{1}$ e da $\mathrm{RP}_{\mathrm{c}}$ na área do pomar. Visto que o coeficiente linear não foi significativamente diferente de zero tanto para a $\mathrm{RP}_{1}$ e para a $\mathrm{RP}_{\mathrm{c}}$ (Tabela 2), a análise de regressão foi efetuada novamente, desta vez desconsiderando-se o coeficiente linear.

Tabela 2. Resultados da análise de regressão linear da $\sigma_{P}$ em função da $\mathrm{RP}_{1} \mathrm{e}$ da $\mathrm{RP}_{\mathrm{c}}$

\begin{tabular}{|c|c|c|c|c|}
\hline Parâmetros & Valor estimado & Erro Padrão & Valor $\mathrm{t}$ & $\operatorname{Pr}>t$ \\
\hline & \multicolumn{4}{|c|}{$\mathrm{RP}_{1}$} \\
\hline A & - 2,585 & 9,381 & $-0,28$ & 0,784 \\
\hline \multirow[t]{2}{*}{ B } & 0,059 & 0,004 & 14,67 & $<0,0001$ \\
\hline & \multicolumn{4}{|c|}{$\mathrm{RP}_{\mathrm{c}}$} \\
\hline A & 11,579 & 7,575 & 1,53 & 0,135 \\
\hline B & 0,053 & 0,003 & 16,58 & $<0,0001$ \\
\hline
\end{tabular}

$\mathrm{RP}_{\mathrm{l}}=$ resistência do solo à penetração medida em laboratório $(\mathrm{kPa}) ; \mathrm{RP}_{\mathrm{c}}=$ resistência do solo à penetração avaliada em campo $(\mathrm{kPa})$

Os valores da pressão de preconsolidação $\left(\sigma_{\mathrm{p}}\right)$ versus $\mathrm{RP}_{1}$ e $\mathrm{RP}_{\mathrm{c}}$ na área de citrus são mostrados na Figura 3. Há uma relação significativa positiva e linear entre a RP e a $\sigma_{\mathrm{P}}$, ou seja um incremento da RP esteve associado a valores superiores de $\sigma_{\mathrm{P}}$, o que corrobora com os resultados apresentados por Culley \& Larson (1987) e Mosaddeghi et al. (2003).

Considerando que avaliações da $\mathrm{RP}_{1}$ apresentam uma maior precisão e que ambas as equações de regressão são semelhantes pode-se assumir que a $\sigma_{\mathrm{P}}$ pode ser corretamente estimada a partir da $\mathrm{RP}_{\mathrm{c}}$ (Figura 3).

Nesse estudo, a relação RP: $\sigma_{P}$ não foi de 10:1, como apresentada por Canarache et al. (2000) e Mosaddeghi et al. (2003). A relação encontrada entre RP: $\sigma_{P}$ foi de 17:1 tanto para a $\mathrm{RP}_{1}$ e $\mathrm{RP}_{\mathrm{c}}$. 
O motivo da diferença apresentada neste estudo pode estar relacionada com a textura do solo. O teor de argila exerce efeito sobre o comportamento compressivo dos solos (McBride, 1989). Solos com predomínio da fração argila possuem maior capacidade de suporte do solo do que aqueles com predominância da fração areia. A influência da argila na capacidade de suporte de carga do solo está relacionada com a força de coesão entre as partículas. A força de coesão impede a separação e o deslocamento das partículas, aumentando a capacidade de suporte do solo o que corrobora os resultados apresentados por Imhoff et al. (2004).

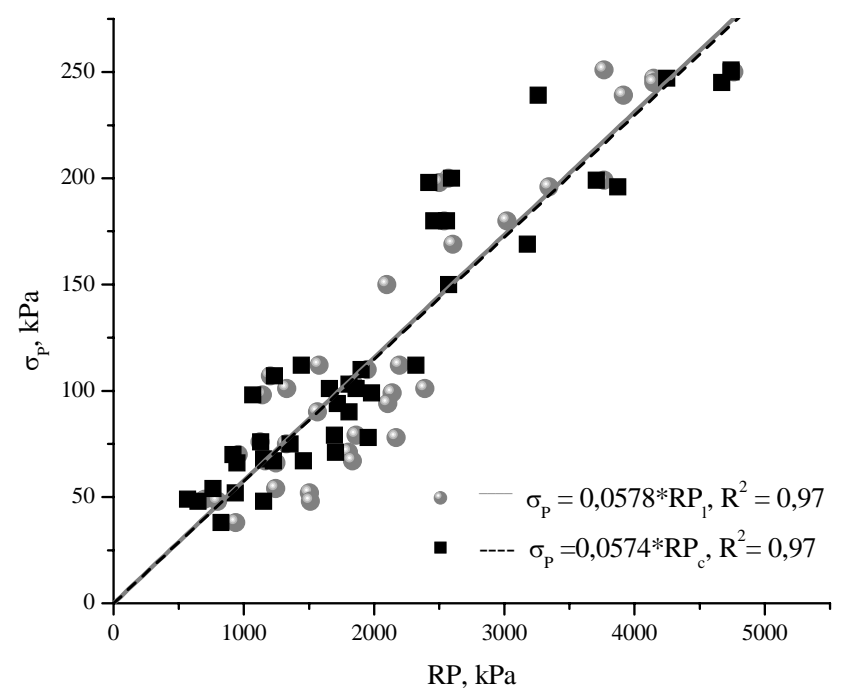

Figura 3 - Pressão de preconsolidação $\left(\sigma_{\mathrm{p}}\right)$ versus resistência do solo à penetração medida em laboratório $\left(\mathrm{RP}_{\mathrm{l}}\right)$ e à campo $\left(\mathrm{RP}_{\mathrm{c}}\right)$ na área de citrus

Os resultados indicam o potencial da utilização da medida da $\mathrm{RP}_{\mathrm{c}}$ para estimar a capacidade de suporte em solos tropicais. Estudos adicionais são necessários para verificar a relação existente entre RP: $\sigma_{P}$ em outros tipos de solos.

\subsection{Conclusões}

Os resultados comprovam a hipótese de que a capacidade de suporte pode ser estimada a partir de avaliações da resistência do solo à penetração em solos de clima 
tropical. Houve uma relação significativa, positiva e linear entre a pressão de preconsolidação e a resistência do solo à penetração medida no laboratório e no campo. A similaridade entre ambas as funções permite afirmar que a pressão de preconsolidação pode ser adequadamente estimada a partir de uma propriedade do solo facilmente mensurável como é a resistência do solo à penetração medida diretamente no campo. 


\section{CONCLUSÕES GERAIS}

No presente estudo confirmou-se a hipótese de que o processo de compactação dos solos de regiões tropicais é influenciado diferenciadamente pelo sistema de manejo aplicado, devido às alterações que o mesmo induz nas propriedades físicas como densidade, aeração e resistência do solo à penetração.

As curvas de compressão uniaxial e o parâmetro pressão de preconsolidação foram influenciados pela intensidade do tráfego de máquinas sendo de grande utilidade na avaliação da heterogeneidade da compactação de um Latossolo Vermelho amarelo sob cultivo de laranja.

O processo de compactação de um Argissolo Vermelho sob sistema pastoril e os valores de pressão de preconsolidação atingidos foram condicionados, pela técnica aplicada no sistema de pastejo rotacionado intensivo para obter maior produtividade da pastagem: a irrigação do solo. A técnica da irrigação deve ser utilizada com cuidado para evitar a degradação do solo.

A resistência do solo à penetração é indicada como uma propriedade física de grande potencialidade na estimativa da capacidade de suporte de carga de um Latossolo Vermelho amarelo sob pomar de laranja.

Pesquisas adicionais serão necessárias para uma melhor compreensão do processo de compactação, principalmente em outros tipos de manejo e de solos desenvolvidos sob clima tropical. 


\section{REFERÊNCIAS BIBLIOGRÁFICAS}

ABAYE, A. O.; ALLEN, V. G.; FONTENOT, J. P. Grazing sheep and cattle together or separately: Effect on soils and plants. Agronomy Journal, v.89, p.380-386, 1997.

ABECITRUS. Associação Brasileira dos Exportadores de Cítricos. 2003. http://www.abecitrus.com.br/safrano.html (16 Jan. 2004).

ALAKUKKU, L.; WEISSKOPF, P.; CHAMEN, W. C. T; TIJINK, F. G. J.; VAN DER LINDEN, J. P.; PIRES, S.; SOMMER, C.; SPOOR, G. Prevention strategies for field traffic-induced subsoil compaction: a review. Part I - Machine/soil interactions. Soil and Tillage Research, v.73, p.145-160, 2003.

ALEXANDROU, A.; EARL, R. The relationship among the pre-compaction stress, volumetric water content and initial dry bulk density of soil. Journal of Agricultural Engineering Research, v.71, p.75-80, 1998.

ANDREWS, H. P.; SNEE, R. D.; SARNER, M. H. Graphical display of means. The American Statistician, n.4, v.34, p.195-199, 1980.

ASSOULINE, S. Modeling soil compaction under uniaxial compression. Soil Science Society of America Journal, v.66, p.1784-1787, 2002.

ASSOULINE, S.; TAVARES FILHO, J.; TESSIER, D. Effects of compaction on soil physical and hydraulic properties: Experimental results modeling. Soil Science Society of America Journal, v.61, p.390-398, 1997.

BAILEY, A. C.; JOHNSON, C. E.; SCHAFER, R. L. A model for agricultural soil compaction. Journal of Agricultural Engineering Research, v.33, n.33, p.257262, 1986. 
BELL, M. J.; BRIDGE, B. J.; HARCH, G. R.; ORANGE, D. N. Physical rehabilitation of degraded krasnozems using ley pastures. Australian Journal of Soil Research, v.35, p.1093-1113, 1997.

BETTERIDGE, K.; MACKAY, A. D.; SHEPHERD T. G.; BARKER, D. J.; BUDDING, P. J.; DEVANTIER, B. P.; COSTALL, D. A. Effect of cattle and sheep treading on surface configuration of a sedimentary hill soil. Australian Journal of Soil Research, v.37, p.743-760, 1999.

BLAKE, G. R.; HARTGE, K. H. Bulk density. In: KLUTE, A. (Ed.). Methods of soil analysis: Physical and Mineralogical Methods. 2.ed. Madison: American Society of Agronomy, Soil Science Society of America, 1986a. cap. 13, p.363-375.

BLAKE, G. R.; HARTGE, K. H. Particle density. In: KLUTE, A. (Ed.). Methods of soil analysis: Physical and Mineralogical Methods. 2.ed. Madison: American Society of Agronomy, Soil Science Society of America, 1986b. cap. 14, p.377-381.

BOONE, F. R.; VERMEULEN, G. D.; KROESBERGEN, B. The effect of mechanical impedance and soil aeration as affected by surface loading on the growth of peas. Soil and Tillage Research, v.32, p.237-251, 1994.

BURGUER, N.; LEBERT, M.; HORN, R. Prediction of the compressibility of arable land. Catena suppl., v.11, p.141-151, 1988.

BUSSCHER, W. J.; BAUER, P. J.; CAMP, C. R.; SOJKA, R. E. Correction of cone index for soil water content differences in a coastal plain soil. Soil and Tillage Research, v.43, p.205-217, 1997.

CANARACHE, A.; HORN, R.; COLIBAS, I. Compressibility of soils in a long term field experiment with intensive deep ripping in Romania. Soil and Tillage Research, v.56, p.185-196, 2000.

CASAGRANDE, A. The determination of the pre-consolidation load and its practical significance. In: INTERNATIONAL CONFERENCE ON SOIL MECHANICS AND FOUNDATION ENGINEERING. Cambridge, 1936. Proceedings. Cambridge: MA Harvard University, 1936. D-34, p. 60-64. 
CHANASYK, D. S.; NAETH, M. A. Grazing impacts on bulk density and soil strength in the foothills fescue grasslands of Alberta, Canada. Canadian Journal of Soil Science, v.75, p. 551-557, 1995.

CULLEY, J. L. B.; LARSON, W. E. Susceptibility to compression of a clay loam Haplaquoll. Soil Science Society of America Journal, v.51, p.562-567, 1987.

DAWIDOWSKI, J. B.; MORRISON, J. E.; SNIEG, M. Measurement of soil layer strength with plate sinkage and uniaxial confined methods. Transactions of the ASAE, v.4, n.5, p.1059-1064, 2001.

DEFOSSEZ, P.; RICHARD, G. Models of soil compaction due to traffic and their evaluation. Soil and Tillage Research, v.67, p.41-64, 2002.

DIAS JÚNIOR, M. S.; PIERCE, F. J. A simple procedure for estimating preconsolidation pressure from soil compression curves. Soil Technology, v.8, p.139-151, 1995.

DIAS JÚNIOR, M. S.; PIERCE, F. J. O processo de compactação do solo e sua modelagem. Revista Brasileira de Ciência do Solo, v.20, p.175-182, 1996.

EARL, R. Assessment of behavior of field soils during compression. Journal of Agricultural Engineering Research, v.68, p.147-157, 1997.

EMPRESA BRASILEIRA DE PESQUISA AGROPECUÁRIA. Centro Nacional de Pesquisa de Solos. Sistema Brasileiro de Classificação de Solos. Brasília: Embrapa Produção de Informação, Rio de Janeiro: Embrapa Solos, 1999. 412p.

FARREL, D. A.; GREACEN, E. L. Resistance to penetration of fine probes in compressible soil. Australian Journal of Soil Research, v.4, p.1-17, 1966.

FEBO, P.; PESSINA, D. Ground pressures exerted by agricultural machines. In: PAGLIAI, M.; JONES, R. (Ed.). Sustainable Land Management Environmental Protection. Advances in Geoecology. Reiskirchen: Catena Verlag, 2002. v.35, cap.4, p.339-350.

FERRERO, A. F. Effect of compaction simulating cattle trampling on soil physical characteristics in woodland. Soil and Tillage Research, v.19, p. 319-329, 1991. 
FLOWERS, M. D.; LAL, R. Axle load and tillage effects on soil physical properties and soybean grain yield on a mollic ochraqualf in northwest Ohio. Soil and Tillage Research, v.48, p.21-35, 1998.

FRITTON, D. D. An improved empirical equation for uniaxial soil compression for a wide range of applied stresses. Soil Science Society of America Journal, v.65, p.678-684, 2001.

GERARD, C. J.; SEXTON, P.; SHAW, G. Physical factors influencing soil strength and root growth. Agronomy Journal, v.74, p.875-879, 1982.

GÉRIK, T. J.; MORRISON JÚNIOR., J. E.; CHICHESTER, F. W. Effects of controlled-traffic on soil physical properties and crop rooting. Agronomy Journal, v.79, p.434-438, 1987.

GREACEN, E. L.; SANDS, R. Compaction of forest soils. A review. Australian Journal of Soil Research, v.18, p.163-189, 1980.

GUPTA, S. C.; ALLMARAS, R. R. Models to assess the susceptibility of soils to excessive compaction. Advances in Soil Science, v.6, p.5-10, 1987.

GYSI, M. Compaction of a Eutric Cambisol under heavy wheel traffic en Switzerland: Field data and a critical state soil mechanics model approach. Soil and Tillage Research, v.61, p.133-142, 2001.

HÄKANSSON, I.; VOORHEES, W. B.; RILEY, H. Vehicle and wheel factors influencing soil compaction and crop response in different traffic regimes. Soil and Tillage Research, v.11, p. 239-282, 1988.

HARTE, K.K. The effect of soil deformation on physical soil properties: A discourse on the common background. In: HORN, R.; AKKER Van Den, J.J.H.; ARVIDSSON, J. (Ed.) Subsoil compaction: Distribution, processes and consequences. Advances in Geoecology. Reiskirchen: Catena Verlag, 2000. v.32, p.22-31.

HORN, R. Compressibility of arable land. Catena, suppl. v.11, p.53-71, 1988.

HORN, R. Stress-strain effects in structured unsaturated soils on coupled mechanical and hydraulic processes. Geoderma, v.116, p.77-88, 2003. 
HORN, R.; LEBERT, M. Soil compactability and compressibility. In: SOANE, B.D.; VAN OUWERKERK, C. (Ed.) Soil compaction in crop production. Amsterdam: Elsevier, 1994. p.45-69.

IMHOFF, S.; SILVA, A.P. da; FALLOW, D. Susceptibility to compaction, load support capacity and soil compressibility of Hapludox. Soil Science Society of America Journal, v.68, p.17-24, 2004.

JORAJÚRIA, D.; DRAGHI, L.; ARAGON, A. The effect of vehicle weight on the distribution of compaction with depth and the yield of Loliun / Trifolium grassland. Soil and Tillage Research, v.41, p.1-12, 1997.

KARLEN, D. L.; DITZLER, C. A.; ANDREWS, S. S. Soil quality: why and how? Geoderma, v.114, p.145-156, 2003.

KIRBY, J. M.; BENGOUGH, A. G. Influence of soil strength on root growth: experiments and analysis using a critical-state model. European Journal of Soil Science, v.53, p.119-128, 2002.

KLUTE, A. Water retention: Laboratory methods. In: KLUTE, A. (Ed.) Methods of soil analysis: Physical and mineralogical methods. 2.ed. Madison: American Society of Agronomy, 1986. cap. 26, p.635-660.

KONDO, M. K. Compressibilidade de três Latossolos sob diferentes usos. Lavras, 1998. 95p. Dissertação (Mestrado) - Universidade Federal de Lavras.

KONDO, M. K.; DIAS JÚNIOR, M. S. Compressibilidade de três Latossolos em função da umidade e uso. Revista Brasileira de Ciência do Solo, v.23, n.2, p.211218, 1999.

KOOLEN, A. J.; LERINK, P.; KURSTJENS, D. A. G.; AKKER J. J. H. Van Den; ARTS, W. B. M. Prediction of aspects of soil-wheel systems. Soil and Tillage Research, v.24, p.381-396, 1992.

LAMPURLANÉS, J.; CANTERO-MARTÍNEZ, C. Soil bulk density and penetration resistance under different tillage and crop management systems and their relationship with barley root growth. Agronomy Journal, v.95, p.526-536, 2003. 
LARSON, W. E.; GUPTA, S. C. Estimating critical stress in unsaturated soils from changes in pore water pressure during confined compression. Soil Science Society of America Journal, v.44, n.6, p.1127-1132, 1980.

LARSON, W. E.; GUPTA, S. C.; USECHE, R. A. Compression of agricultural soils from eight soil orders. Soil Science Society of America Journal, v.44, n.3, p.450457, 1980.

LEBERT, M.; HORN, R. A method to predict the mechanical strength of agricultural soils. Soil and Tillage Research, v.19, n.2/3, p.274-286, 1991.

LERINK, P. Prediction of the immediate effects of traffic on field soil qualities. Soil and Tillage Research, v.16, p.153-166, 1990.

LHOTSKÝ, J.; BERAN, P. PARIS, P.; VALIGURSKÁ, L. Degradation of soil by increasing compression. Soil and Tillage Research, v.19, p.287-295, 1991.

LIPIEC, J.; HATANO, R. Quantification of compaction effects on soil physical properties and crop growth. Geoderma, v.116, p.107-136, 2003.

MACNABB, D. H.; BOERSMA, L. Evaluation of the relationship between compressibility and shear strength of Andisols. Soil Science Society of America Journal, v.57, p.923-929, 1993.

MACNABB, D. H.; BOERSMA, L. Nonlinear model for compressibility of partly saturated soils. Soil Science Society of America Journal, v.60, p.333-341, 1996.

MAPFUMO, E.; CHANASYK, D.S.; NAETH, M.A.; BARON, V.S. Soil compaction under grazing of annual and perennial forages. Canadian Journal of Soil Science, v.79, p.191-199, 1999.

MATHSOFT. Mathcad professional reference manual. Cambridge, MA: Mathsoft, 2000. 180p.

MCBRIDE, R. A. Estimation of density-moisture-stress functions from uniaxial compression of unsaturated, structured soils. Soil and Tillage Research, v.19, p.383-397, 1989.

MCBRIDE, R. A.; JOOSSE, P. J. Overconsolidation in agricultural soil: Pedotransfer functions for estimating preconsolidation stress. Soil Science Society of America Journal, v.60, p.373-380, 1996. 
MCNABB, D. H.; STARTSEV, A. D.; NGUYEN, H. Soil wetness and traffic level effects on bulk density and air-filled porosity of compacted boreal forest soils. Soil Science Society of America Journal, v.65, p.1238-1247, 2001.

MORENO, J. H. N.; GASCON, B. V. Effect of soil condition on orange trees. Communication in Soil Science and Plant Analysis, v.25, n.9/10, p.1747-1753, 1994.

MOSADDEGHI, M. R.; HAJABBASI, M. A.; HEMMAT, A.; AFYUNI, M. Soil compactibility as affected by soil moisture content and farmyard manure in central Iran. Soil and Tillage Research, v.55, p.87-97, 2000.

MOSADDEGHI, M. R.; HEMMAT A.; HAJABBASI, M. A.; ALEXANDROU, A. Pre-compression stress and its relation with the physical and mechanical properties of a structurally unstable soil in central Iran. Soil and Tillage Research, v.70, p.53-64, 2003.

NEVENS, F.; REHEUL, D. The consequences of wheel-induced soil compaction and subsoiling for silage maize on a sandy loam soil in Belgium. Soil and Tillage Research, v.70, p.175-184, 2003.

NIE, Z. N.; WARD, G. N.; MICHAEL, A. T. Impact of pugging by dairy cows on pastures and indicators of pugging damage to pasture soil on south -western Victoria. Australian Journal Agricultural of Soil Research, v.52, p.37-43, 2001.

OLIVEIRA, G. C. de; DIAS JÚNIOR, M. de S.; RESCK, D. V. S.; CURI, N. Alterações estruturais e comportamento compressivo de um Latossolo Vermelho distrófico argiloso sob diferentes sistemas de manejo. Pesquisa Agropecuária Brasileira, v.38, p.291-299, 2003.

OLIVEIRA, M. T.; MERWIN, I. A. Soil physical conditions in a New York orchard after eight years under different groundcover management systems. Plant and Soil, v.234, p.233-237, 2001.

PRADO, R. de M.; ROQUE, C. G.; SOUZA, Z. M. de. Sistemas de preparo e resistência à penetração e densidade de um Latossolo Vermelho eutrófico em cultivo intensivo e pousio. Pesquisa Agropecuária Brasileira, v.37, n.12, p.1795,1801, 2002. 
PROFFITT, A. P. B.; BENDOTTI, S.; HOWELL, M. R.; EASTHAM, J. The effect of sheep trampling and grazing on soil physical properties and pasture growth for a Red-Brown earth. Australian Journal Agricultural of Soil Research, v.44, p.317331, 1993.

PYTKA, J.; DABROWSKI, J. Determination of stress-strain relationship for sandy soil in field experiments. Journal of Terramechanics, v.38, p.185-200, 2001.

RAGHAVAN, G. S. V.; ALVO, P.; MCKYES, E. Soil compaction in agriculture: A view toward managing the problem. Advances in Soil Science, v.11, p.1-35, 1990.

RAJARAM G.; ERBACH, D. C. Drying stress effect on mechanical behavior of a clay loam soil. Soil and Tillage Research, v.49, p.147-158, 1998.

RÖMKENS, M. J. M.; MILLER, R. D. Predicting root size and frequency from onedimensional consolidation data - A mathematical model. Plant and Soil, v.35, p.237-248, 1971.

SALIRE, E. V.; HAMMEL, J. E.; HARDCASTLE, J. H. Compression of intact subsoils under short-duration loading. Soil and Tillage Research, v. 31, p.235-248, 1994.

SANChES, A. C.; SILVA, A. P. da; TORMENA, C. A.; RIGOLIM, A. T. Impacto do cultivo de citrus em propriedades químicas, densidade do solo e atividade microbiana de um Podzólico Vermelho-Amarelo. Revista Brasileira de Ciência do Solo, v.23, n.1, p.91-99, 1999.

SÁNCHES-GIRÓN, V.; ANDREU, E.; HERNANZ, J. L. Response of five types of soil to simulated compaction in the form of confined uniaxial compression test. Soil and Tillage Research, v.48, n.1/2, p.37-50, 1998.

SÁNCHEZ-GIRÓN, V.; ANDREU, E.; HERNANZ, J. L. Stress relaxation of five different soil samples when uniaxially compacted at different water contents. Soil and Tillage Research, v.62, p.85-99, 2001.

SILVA, A. P. da; IMHOFF, S.; CORSI, M. Evaluation of soil compaction in an irrigated short-duration grazing system. Soil and Tillage Research, v.70, p.83-90, 2003. 
SILVA, V. R.; REINERT, D. J.; REICHERT, J. M. Susceptibilidade à compactação de um Latossolo Vermelho Escuro e de um Podzólico Vermelho Amarelo. Revista Brasileira de Ciência do Solo, v.24, n.2, p.239-249, 2000.

SILVA, V. R.; REINERT, D. J.; REICHERT, J. M.; SOARES, J. M. Fatores controladores da compressibilidade de um Argissolo Vermelho Amarelo distrófico arênico e de um Latossolo Vermelho distrófico típico. I- Estado inicial de compactação. Revista Brasileira de Ciência do Solo, v.26, p.1-8, 2002.

SMITH, C. W.; JOHNSTON, M. A.; LORENTS, S. Assessing the compaction susceptibility of South African forestry soils. II - Soil properties affecting compactibility and compressibility. Soil and Tillage Research, v.43, n.3/4, p.335354, 1997.

SNAKIN, V. V.; KRECHETOV, P. P.; KUZOVNIKOVA, T. A. The system of assessment of soil degradation. Soil Techonology, v.8, p.331-343, 1996.

SOANE, B.D. Process of soil compaction under vehicular traffic and means of alleviating it. In: LAL, R.; SANCHEZ, P.A.; CUMMINGS, R.W. (Ed.) Land clearing and development in the tropics. Rotterdam: Balkema Publ., 1986. p.265297.

SOANE, B. D. The role organic matter in soil compactibility: A review of some practical aspects. Soil and Tillage Research, v.16, p.179-201, 1990.

SOANE, B. D.; VAN OUWERKERK, C. Soil compaction in crop production. In: SOANE, B. D.; VAN OUWERKERK, C. (Ed.). Soil compaction problems in world agriculture. Elsevier: Amsterdan, 1994. p.1-21.

SOANE, B. D.; BLACKWELL, P. S.; DICKSON, J. W. Compaction by agricultural vehicles: A review. II - Compaction under tyres and other running gear. Soil and Tillage Research, v.1, n.4, p.373-400, 1981.

SOANE, B. D.; DICKSON, J. W.; CAMPBELL, D. J. Compaction by agricultural vehicles: A review. III- Incidence and control of compaction in crop production. Soil and Tillage Research, v.2, p.3-36, 1982.

STATISTICAL ANALYSIS SYSTEM INSTITUTE. SAS/STAT procedure guide for personal computers. 5.ed. Cary: SAS Institute, 1991. 1104p. 
STEPHENSON, G. R.; VEIGEL, A. Recovery of compacted soil on pastures used for winter cattle feeding. Journal of Range Management, v.40, n.1, p.46-48, 1987.

STOLF, R. A compactação do solo e perspectivas da subsolagem em citros. Laranja, v.2, n.8, p.283-308, 1987.

TANNER, C. B.; MAMARIL, C. P. Pasture soil compaction by animal traffic. Agronomy Journal, v.51, p.329-331, 1959.

TAYLOR, H. M.; ROBERSON, G. M.; PARKER JÚNIOR, J. J. Soil strength-root penetration relations to medium to coarse-textured soil materials. Soil Science, v.102, n.1, p.18-22, 1966.

TERSI, F. E. A.; ROSA, S. M. A subsolagem no manejo de solo para os pomares de citros. Laranja, v.16, n.2, p.289-298, 1995.

TWERDOFF, D. A.; CHANASYK, D. S.; MAPFUMO, E.; NAETH, M. A.; BARON, V. S. Impacts of forage grazing and cultivation on near-surface relative compaction. Canadian Journal of Soil Science, v.79, p.465-471, 1999.

VAZ, C. M. P.; HOPMANS, J. W. Simultaneous measurement of soil penetration resistance and water content with a combined penetrometer-TDR moisture probe. Soil Science Society America Journal, v.65, p.4-12, 2001.

VEEN, B. W.; BOONE, F. R. The influence of mechanical resistance and soil water on the growth of seminal roots of maize. Soil and Tillage Research, v.16, p.219-226, 1990.

VEENHOF, D. W.; MCBRIDE, R. A. Overconsolidation in agricultural soils: I Compression and consolidation behavior of remolded and structured soils. Soil Science Society of America Journal, v.60, p.362-373, 1996.

WILLATT, S. T.; PULLAR, D. M. Changes in soil physical properties under grazes pastures. Australian Journal of Soil Research, v.22, p.343-348, 1983.

WU, L.; FENG, G.; LETEY, J.; FERGUSON, L.; MITCHELL, J.; MCCULLOUGHSANDEN, B.; MARKEGARD, G. Soil management effects on the nonlimiting water range. Geoderma, v.114, p.401-414, 2003. 
ZHANG, H.; HARTGE, K. H.; RINGE, H. Effectiveness of organic matter incorporation in reducing soil compactability. Soil Science Society of America Journal, v. 61, p.239-245, 1997.

ZIMMER, A.; SILVA, M. P.; MAURO, R. Sustentabilidade e impactos ambientais da produção animal em pastagens. In: PEIXOTO, A. M; MOURA, J. C.; PEDREIRA, C. G. S.; FARIA, V. P. Inovações tecnológicas no manejo de pastagens. Piracicaba: FEALQ, 2002. cap.2, p.31-58. 